\title{
The Cif proteins from Wolbachia prophage WO modify sperm genome integrity to establish cytoplasmic incompatibility
}

Short title: The cytoplasmic incompatibility proteins modify sperm genome integrity Authors: Rupinder Kaur ${ }^{1,2, *}$, Brittany A. Leigh ${ }^{1,2, *}$, Isabella T. Ritchie ${ }^{1,2}$ and Seth R.

Bordenstein ${ }^{1,2,3,4,5}$

\section{Affiliations:}

${ }^{1}$ Vanderbilt University, Department of Biological Sciences, Nashville, TN, USA

${ }^{2}$ Vanderbilt University, Vanderbilt Microbiome Innovation Center, Nashville, TN, USA

${ }^{3}$ Vanderbilt University Medical Center, Department of Pathology, Microbiology \& Immunology, Nashville, TN, USA

${ }^{4}$ Vanderbilt University Medical Center, Vanderbilt Institute for Infection, Immunology and Inflammation, Nashville, TN, USA

${ }^{*}$ Co-first authors

${ }^{5}$ Correspondence to:

Seth R. Bordenstein, Nashville, TN, 37235, Phone 615.322.9087, e-mail

$\underline{\text { s.bordenstein@vanderbilt.edu }}$ 


\section{Abstract}

Inherited microorganisms can selfishly manipulate host reproduction to drive through populations. In Drosophila melanogaster, germline expression of the native Wolbachia prophage WO proteins CifA and CifB cause cytoplasmic incompatibility (CI) in which embryos from infected males and uninfected females suffer catastrophic mitotic defects and lethality; however, in infected females, CifA expression rescues the embryonic lethality and thus imparts a fitness

.
advantage to the maternally-transmitted Wolbachia. Despite widespread relevance to sex determination, evolution, and vector control, the mechanisms underlying when and how CI impairs male reproduction remain unknown and a topic of debate. Here we use cytochemical, microscopic, and transgenic assays in D. melanogaster to demonstrate that CifA and CifB proteins of $w \mathrm{Mel}$ localize to nuclear DNA throughout the process of spermatogenesis. Cif proteins cause abnormal histone retention in elongating spermatids and protamine deficiency in mature sperms that travel to the female reproductive tract with Cif proteins. Notably, protamine gene knockouts enhance wild type CI. In ovaries, CifA localizes to germ cell nuclei and cytoplasm of early-stage egg chambers, however Cifs are absent in late-stage oocytes and subsequently in fertilized embryos. Finally, CI and rescue are contingent upon a newly annotated CifA bipartite nuclear localization sequence. Together, our results strongly support the Host 
Numerous animal species harbor heritable microorganisms that alter host fitness in beneficial and harmful ways. The most common, maternally-inherited bacteria are Wolbachia that typically reside intracellularly in reproductive tissues of both male and female arthropods. Here, they induce reproductive modifications with sex specific effects such as cytoplasmic incompatibility (CI) that can selfishly drive the bacteria to high frequencies in host populations. strategies [4-9] by causing lethality of embryos from Wolbachia-infected males and uninfected females. As CI is rescued by Wolbachia-infected females with the same strain [10,11], the phenotype accordingly imparts a relative fitness advantage to infected females that transmit the bacteria [12].

\section{Two genes, cytoplasmic incompatibility factors cifA and cifB, occur in Wolbachia} prophage WO within the eukaryotic association module enriched for arthropod functions and homology [13-15]. We previously demonstrated that dual, transgenic expression of $c$ ifA and $c$ if $B$ from w Mel Wolbachia in Drosophila melanogaster males induces CI, while single expression of

43 of embryonic development. Normally after fertilization, the sperm-bound 'protamines' are

44 removed in the embryo and replaced by maternally supplied 'histones', resulting in the rapid 45 remodeling of the paternal chromatin [20]. However, during CI, there is a delay in the deposition 46 of maternal histones onto the paternal chromatin, resulting in altered DNA replication, failed 
chromosome condensation, and various mitotic defects that generate embryonic death [13,2127].

The incipient, pre-fertilization events in the reproductive tissues that establish CI and rescue remain enigmatic and under recent debate, namely whether (i) Cifs modify the paternal genome during spermatogenesis (Host-Modification model) or embryogenesis (Toxin-Antidote model) and (ii) rescue occurs or does not occur by CifA binding CifB in the embryo [28,29]. These two key observations can conclusively differentiate the mechanistic models of CI, though they have not been explicitly addressed to date. Notably, paternal transmission of the proteins from sperm to embryo may occur under either mechanistic model $[11,29]$. Recent work proposed the Toxin-Antidote model is operational using transgenic, heterologous expression of Cif proteins from $w$ Pip Wolbachia [30]. In this study, the $\mathrm{CifB}_{w \text { Pip }}$ protein paternally transfers to the fly embryo and associates with DNA replication stress of the paternal genome in the embryo. However, without the ability to rescue this non-native, transgenic CI and thus visualize CifACifB binding in the rescue embryo, these interesting results do not yet resolve the predictions of the two models. Additionally, the paternal DNA replication defects observed in the embryos may be established before fertilization by the Cif proteins, in concordance with Host Modification model. melanogaster gametogenesis and embryogenesis and then perform genome integrity measurements of developing sperm across transgenic, mutant, and wild type treatment groups. We describe the following cell biological and gametic chromatin events underpinning the Host Modification model of CI and rescue: (i) CifA and CifB proteins localize to the developing sperm nuclei from early spermatogonium stage to late elongating spermatids; (ii) In mature 
sperm, CifA associates with sperm tails and occasionally occurs in the acrosome, whereas CifB localizes to the acrosome in all mature sperms; (iii) Cifs increase histone retention in developing spermatids and decrease protamine levels in mature sperms; (iv) Both CI and rescue are dependent upon a newly annotated bipartite nuclear localization signal (bNLS) in CifA that impacts nuclear localization and sperm protamine levels;

(v) During copulation, both Cif proteins transfer with the mature sperm exhibiting reduced protamine levels; importantly, protamine mutant flies enhance wild type CI; (vi) In the ovaries, CifA is cytonuclear in germline stem cells and colocalizes with Wolbachia in the nurse cell cytoplasm; (vi) CifA is absent in the embryos, and thus rescue must be established in oogenesis independently of CifA's presence in the embryo. Taken together, results demonstrate that prophage WO-encoded Cif proteins from Wolbachia invade gametic nuclei to modify chromatin integrity at the histone to protamine transition stage in males. At the mechanistic level, results support the Host Modification model of CI and rescue whereby native Cifs wield their impacts prior to fertilization.

\section{Results}

\section{CifA and CifB invade sperm nuclei during spermatogenesis and spermiogenesis}

To evaluate the cellular localization of the Cif proteins, we generated monospecific polyclonal antibodies for visualizing the proteins in reproductive tissues (Fig S1). In $D$. melanogaster males, the sperm morphogenesis process is subdivided into two events (i) Spermatogenesis including mitotic amplification and meiotic phases, and (ii) Spermiogenesis, a post-meiotic phase. During spermatogenesis, the germline stem cell undergoes four rounds of synchronous mitotic divisions to produce 16 precursor cells called spermatogonia. The spermatogonia then grow and become spermatocytes [31]. After the growth phase, the 
93 spermatocytes divide by meiosis and differentiate into 64 haploid round onion spermatids. Post-

94 meiosis, the round sperm nuclei elongate to gradually change their shape accompanied by

95 reorganization of the chromatin during the canoe stage [32]. This results in an individualization

96 complex forming slim, needle-shaped sperm nuclei with reduced volume [31,32]. Elongation and

97 individualization of the spermatids is the final stage of spermiogenesis, after which the mature

98 sperms are transported to the seminal vesicle [31,33].

99 CifA, but not CifB, localizes in the germline stem cells at the apical end of testes in $<8 \mathrm{hr}$

$100 \quad$ old cifA and cifB transgene-expressing (Fig 1) and wild type wMel+ males (Fig S2). CifA and

101 CifB were both detected in the nuclei of mitotic spermatogonium and spermatocytes. CifA is

102 more abundant than CifB in the spermatogonium stage (Fig S3A, Table S1). In the post-meiotic,

103 round onion spermatids, clusters of both CifA and CifB are adjacent to the nuclei. In the

104 elongating canoe-shaped spermatids, CifA and CifB localize apical to the sperm head nucleus, in

105 what is likely the acrosome (Fig 1, Fig S2). CifB is present in all of the spermatid nuclei,

106 whereas CifA is present on average in $39 \%$ of the elongating spermatids per sperm bundle (Fig

107 S3B). During the elongating canoe stage, chromatin-bound histones are typically removed and

108 replaced with protamines to yield compact nuclear packaging and chromatin organization of

109 sperm DNA [32]. After nuclear compaction is complete, neither of the Cif proteins are detectable

110 in late spermatid needle-shaped nuclei (Fig 1) and in the mature sperms from the seminal vesicle

111 (Fig S4), indicating either the Cif proteins are fully stripped, or they might not be accessible by

112 the antibodies when the chromatin is tightly compacted [34,35]. To evaluate Cif

113 presence/absence in the mature sperm, we decondensed sperms after isolations from seminal

114 vesicles of $<8 \mathrm{hr}$ old males (see methods) and stained them with the respective Cif antibodies.

115 CifA is common along sperm tails in a speckled pattern (Fig 1, Fig S2) and infrequently present 
116 in the acrosome region, on average in $45 \%$ or $0 \%$ of the mature sperm heads depending upon the

117 sampled seminal vesicles (Fig S3B). CifB is present in all of the acrosomal tips of the sperms

118 and not localized to the sperm tails (Fig 1, Fig S2).

119 During spermatogenesis, Wolbachia are stripped into the cytoplasmic waste bags, which 120 eliminate excess cytoplasmic material during the process of spermatid elongation [23]. Here, we 121 show that some CifA and CifB proteins are also stripped by the individualization complex into 122 the cytoplasmic waste bag (Fig S5). Since Wolbachia are not present in the mature sperms $123[36,37]$, these data suggest that the Cif proteins exit Wolbachia cells during spermatogenesis to 124 possibly interact with and modify sperm DNA (see below). Taken together, these findings 125 demonstrate CifA and CifB proteins access Drosophila sperm nuclei throughout development.

Since the Cifs localize to developing sperm nuclei during spermatogenesis, we 130 integrity - a central prediction of the Host Modification model of CI [11,29]. At the histone-to131 protamine transition stage during spermatogenesis [38], histones normally undergo various post132 translational modifications (PTMs) for removal and replacement by smaller protamines for tight 133 chromatin reorganization [38-41]. Lack of PTMs can lead to histone-bound chromatin with 134 improper protamine deposition that causes paternal chromatin defects, male infertility, and 135 embryonic lethality $[27,42,43]$. Thus, the incipient defects initiated in the testes can lead to post136 fertilization catastrophes. bundles at the late canoe stage in CI- and non-CI causing males. We detected significantly 
139 increased histone retention in both $w \mathrm{Mel}+$ and cifAB-expressing testes from $<8$ hr old males 140 compared to the negative controls (Fig 2A, Fig S6, Table S1). Single transgenic expression 141 showed significantly less histone-retaining bundles at this stage similar to $w$ Mel- negative 142 controls (Fig S7A, Table S1). To detect if abnormal histone retention is linked with protamine 143 deficiency in mature sperms, we next used the fluorochrome chromomycin A3 (CMA3) stain 144 that fluoresces upon binding to protamine-deficient regions of DNA $[44,45]$. Mature sperms 145 isolated from wild-type seminal vesicles of young (high CI-inducing) $w$ Mel+ males exhibit 146 increased protamine deficiency relative to $w$ Mel- males (Fig 2B, Table S1).

147 To investigate if lack of protamines associates with CI, we isolated sperms from $<8 \mathrm{hr}$ old 148 males with a protamine A and B knockout mutant line. We show that protamine mutants, both in 149 the presence $(\Delta$ Prot +$)$ and absence ( $\Delta$ Prot-) of Wolbachia, also exhibit a significant increase in 150 fluorescence relative to $w$ Mel- (Fig 3A, Table S1). Moreover, a key outcome of higher protamine 151 deficiency in $\Delta$ Prot+ males with Wolbachia is an increase in CI compared to wild type wMel+ 152 CI, under the same experimental setup (Fig 3B, Table S2). These findings indicate an additive 153 effect by Wolbachia and $\Delta$ Prot knockouts on the protamine deficiency and CI penetrance. 154 Notably, $\Delta$ Prot- males do not recapitulate CI on their own; thus, the protamine deficiency is not 155 the sole cause of CI and must operate in conjunction with other CI modifications. Consistent 156 with these results, 7-day old $w$ Mel+ males that express almost no CI exhibit a similarly weak 157 protamine deficiency level to $w$ Mel- males, as expected (Fig S8A, Fig S8B and Table S2). 158 Moreover, transgene analyses specify both single and dual expression of CifA and CifB cause 159 protamine deficiencies at significantly higher levels than negative controls of $w$ Mel- and a non160 CI transgene (Fig S7B, Table S1). Since singly expressed Cifs do not cause CI in $D$. 
161 melanogaster [16] (Fig S7C, Table S2), additive effects on the protamine deficiency and/or

162 histone retention due to abnormal PTMs may be required to fully establish CI. harbor a predicted bipartite nuclear localization sequence (bNLS) (Table S3) in the most conserved region of the protein $[13,47,48]$ that is under strong purifying selection [17]. As nuclear localization signals bind to the extended surface groove of nuclear transport protein importin- $\alpha$, also known as karyopherin- $\alpha$ [49], we hypothesized that sperm nuclear localization of CifA and CI and rescue are dependent on the bNLS. To test this hypothesis, we mutagenized two bNLS sequences with alanine substitutions (aa189-190 for NLS1 (denoted cifA 189 ), aa224225 for NLS2 (denoted $\left.c i f A_{224}\right)$ ), and we additionally deleted the entire bNLS region ( $\left.c i f A_{\triangle b N L S}\right)$ (Fig 4A). The bNLS deletion also corresponds to the weakly predicted catalase-rel domain in CifA $[47,48]$.

Each bNLS mutant, individually and together ( cif $\left._{189 ; 224}\right)$, was dually expressed in testes with transgenic, intact cifB to assess $\mathrm{CI}$ and singly expressed in females to assess rescue. Transgenic cifA 189 expression significantly reduced CI and rescue as previously reported (Fig 4B,C and Table S2) [48]. Conversely, transgenic cifA 224 expression showed no significant difference from the controls in either CI or rescue, suggesting this region has little to no impact. However, when both mutants are expressed in cif $_{189 ; 224}$ or when the entire bNLS is deleted, CI and rescue are strongly inhibited (Fig 4B,C and Table S2). These results highlight the importance of the nuclear localization sequence in inducing CI as well as rescue. To determine if the lack of CI induction is due to non-nuclear localization of CifA protein, we used the deletion mutant 
$184 \quad c i f A_{\triangle b N L S}$ with wild type $\operatorname{cifB}$ to demonstrate that in contrast to its normal, nuclear localization

185 (Fig 1), it mislocalizes to the cytoplasm of onion stage spermatids rather than the nuclei (Fig 186 4D). Additionally, to test if deletion of the bNLS impacts sperm genomic integrity, we 187 performed CMA3 based protamine-deficiency assay (as described above) and found reduced 188 protamine deficiency levels in matures sperms due to non CI-causing $c i f A_{\triangle b N L S} ; B$ line compared 189 to CI-causing cifAB (Fig 4E, Table S1), providing further evidence that protamine deficiency is 190 linked to CI. Overall, these data provide previously unknown findings that a functional nuclear 191 localization sequence and CifA nuclear localization impacts CI, rescue, and sperm protamine 192 levels.

To investigate if Cif proteins and/or the genome integrity modifications transfer 196 paternally to the female reproductive tract, single male:female pairwise matings were set up for 197 CI and rescue crosses. After 4 hrs of mating, we isolated the whole uterus (Fig 5A) including the 198 sperm storage organs (spermathecae (SP) and seminal receptacle (SR)). Following sperm 199 decondensation, antibody staining, and microscopy, we observed two key results. First, both 200 CifA and CifB proteins transfer with sperm tails and heads, respectively, to the sperm storage 201 organs of Wolbachia-free females (Fig 5B). Second, the CI-associated sperm protamine 202 deficiency induced by $w \mathrm{Mel}+$ and $c i f A B$-expressing males transfers and persists in the sperms 203 isolated from SP and SR of Wolbachia-free, mated females (Fig 5C,D and Table S1). These 204 findings connect a paternally-transferred sperm modification with the activity of Wolbachia and 205 the Cifs themselves. Results strongly support the Host Modification model of CI since a Cif206 induced sperm modification established in the testes transfers to the female reproductive tract. 
CifA is present in early oogenesis and absent from mature eggs and rescue embryos rescue is unknown and central to further differentiating the mechanistic models of CI. For instance, CifA in females may modify reproductive cell biology to nullify CI-inducing sperm modifications in the embryo (Host-Modification), or alternatively, CifA may directly bind CifB in the embryos to prevent its proposed CI toxicity (Toxin-Antitoxin). Using CifA antibodies, we show in $w \mathrm{Mel}+$ and cifA transgene-expressing ovaries that CifA protein is cytonuclear and specifically localizes to cyst DNA in region 1 of the germarium (Fig 6A), indicative of nuclear access in ovaries similar to that in testes (Fig 1). Cystoblast in the germarium undergoes rounds of mitotic divisions to produce oocyte and nurse cells [50,51]. Along egg chamber stages 2-8 of $w$ Mel+ females, CifA colocalizes with Wolbachia in the nurse cells and oocyte cytoplasm (Fig 6A). While Wolbachia are abundant in the stage 10 egg chamber, CifA is notably absent. In transgenic females, CifA is also primarily detected in the germarium and cytoplasm of the early egg chambers and absent in late egg chambers (Fig 6A, Fig S9A). Presence of high levels of CifA in Wolbachia-infected eggs is proposed to rescue CI; importantly, we did not detect CifA in 30-60 min old rescue embryos during early mitotic divisions (Fig 6B). Moreover, CifA was not with embryonic DNA at both embryonic developmental stages.

CifB from $w$ Pip was recently shown to be paternally-transferred to the CI embryos [30]. We evaluated if $w \mathrm{Mel} \mathrm{CifB}$ colocalizes with mitotic, embryonic DNA after fertilization. CifB is absent from embryonic nuclei and the cytoplasm of CI embryos (Fig 6B), suggesting that CifB is not inherited with the paternal DNA to the embryo. The absence of CifA and CifB in late wild 
230 type and transgenic eggs and embryos indicates that host gametic changes prime the embryo for

$231 \quad$ CI and rescue before fertilization, as predicted by the Host Modification model of CI. This 232 inference is also consistent with previous studies where ovarian, rather than embryonic, 233 transgenic cifA expression rescues CI [13,15-17,52].

\section{Discussion}

At the genetic level, dual expression of cifA and cifB or single expression of cifB can recapitulate CI; and cifA rescues CI [13,15-19,52]. However, the cellular and mechanistic bases of CI and rescue remain unresolved and the subject of several questions: When and where do the Cif proteins localize in testes to potentiate CI? Are the Cifs transferred to the embryo already modified for CI-defining defects? Do CifA and CifB bind in the embryo to rescue lethality? Here, we establish that both CifA and CifB proteins invade nuclei of developing spermatids and modify paternal genome integrity by altering the normal Histone-Protamine transition process. deficiency in CI-causing male gametes to induce CI. Moreover, knocking out protamines enhances wild type CI, and a nuclear localization sequence in CifA is essential for CI, rescue, and protamine deficiency. Finally, binding of CifA and CifB in the embryo is not evident. spermatogenesis, when histones are replaced by protamines [53]. This compaction process is highly conserved from flies to humans [54-56] and plays a crucial role in successful fertilization and embryonic development $[57,58]$. Histone marks are carriers of transgenerational epigenetic information [59], and changes in the sperm epigenome can lead to detrimental consequences including early embryonic lethality and birth defects $[59,60]$. Histones undergo various post- 
translational modifications (PTMs) such as ubiquitination, methylation, phosphorylation and acetylation before degradation and removal from the sperm chromatin [38]. Therefore, abnormally-retained histones could result from aberrant PTMs in Cif-expressing flies. In a protein interactome screen, both ubiquitin and histone $\mathrm{H} 2 \mathrm{~B}$ were determined as binding host candidates to CifB [52]. Thus, it is possible that inhibition of the histone ubiquitination process mediates abnormal histone retention. Additionally, histone acetylation during the canoe stage of spermatogenesis is required for histone eviction in Drosophila [61]. Indeed, reduced acetylation levels lead to abnormal histone retention and protamine deficiency, which causes embryonic inviability in flies [61] and in mammals [40,62,63]. Future research investigating what specific PTMs are altered at the Histone-Protamine transition stage will help elucidate the molecular pathway(s) leading to $\mathrm{CI}$.

The paternally-derived Cif proteins travel with the sperms to the female reproductive tract, where CifB is present in the acrosomal region and CifA occurs along the tail. Notably, both Cifs are not evident in the embryos. While the presence of paternally-transferred Cifs is ambiguous to the mechanistic models of CI [11,29] and recently confirmred in another transgenic study [30], it is the presence of CifA-CifB binding in the rescue embryo that would support the Toxin-Antitoxin model. However, there is no evidence of this binding phenomenon in the embryos to date. Thus, we conclude that Cifs act before fertilization to prime the sperm chromatin and incipiently launch CI. Paternal effect proteins can modify sperm in various systems to bestow embryonic defects, even though the proteins themselves do not transfer to the embryos $[60,64,65]$.

In Drosophila, the sperm enters the egg with an intact membrane [66]. Therefore, absence of Cifs in the embryos raises the question at what point the presence of CifA along the 
tail and CifB in the acrosome is lost before the sperm enters the egg. One possible explanation is that the Cif proteins are released from the sperm upon exocytosis of the acrosome. The acrosome, best known as a secretory vesicle, undergoes exocytosis and releases its contents to facilitate sperm-egg binding in mammals $[67,68]$. Though not well characterized in insects, studies in the house fly Musca domestica suggest loss of the sperm plasma membrane before entry into the egg, followed by exocytosis of acrosomal contents during passage of the sperm through the egg micropyle [69].

CifA is absent in Wolbachia-infected and transgenic embryos, which indicates that rescue is established during oogenesis under the Host Modification model, and CifA thus does not bind and nullify CifB from w Mel Wolbachia in the embryos. Indeed, a CifA mutant in the newly annotated nuclear localization sequence ablates rescue, suggesting access to ovarian nuclei is proteins [70], yet in light of these results here, the CifA-CifB binding may be central to CI induction instead of rescue. Moreover, mutating amino acid sites across the length of the CifA protein, including binding and non-binding residues, generally ablates CI and rescue [48,70]. Thus, it is possible that CifA mutants that ablate rescue do so by altering the protein structure, function, and/or location of ovarian targets to modify, rather than the embryonic binding of CifA to $\mathrm{CifB}$ in vivo. Future work will be important to resolve how CifA primes oogenesis to alter specific cell biological and biochemical events that underpin rescue.

Once fertilization occurs, protamines from the paternal chromatin must be removed and replaced by maternal histones to decondense and activate the chromatin of the developing embryo [71]. Interestingly, post-fertilization delays in maternal H3.3 histone deposition occur in CI embryos [72]. The delay may in part be due to pre-loaded paternal histones or altered paternal 
300 epigenome information leading to mistiming of maternal histone deposition, hence causing CI.

301 Thus, we propose that a genome integrity network involving histones, protamines, and possibly

302 other factors in the gametes may be a common and defining feature underpinning the onset of CI

303 and rescue.

Altogether, discovery of nuclear-targeting Cif proteins in male and female gametes establishes new insights on the early cell biological and biochemical steps that underpin the CI drive system with relevance to arthropod speciation and pest control [11]. In addition to disentangling the reproductive events of the Cif proteins that control gametogenesis and embryogenesis, the evidence specifies that the Cif proteins modify sperm genomic integrity and transfer paternally, but they themselves do not enter and bind each other in the embryo to enable Wolbachia. More generally, as there are no previous reports of prophage proteins invading animal gametic nuclei to impair the histone-protamine transition during spermatogenesis, the findings have implications for expanding an appreciation of prophage-bacteria-eukaryote interactions into the realm of animal reproduction.

\section{Material and Methods}

\section{Cif proteins antibody development}

Conserved amino acid regions of CifA and CifB proteins from w Mel Wolbachia were previously identified [47]. Using these regions, monospecific polyclonal antibodies were commercially generated by Pacific Immunology through injection of three synthesized and conserved short (20 aa) peptides for each protein into rabbits. Sequences of peptides were CysEYFYNQLEEKDKEKKLTE for CifA, and Cys-DENPPENLLSDQTRENFRR for CifB. The 
resulting $\alpha$-CifA and $\alpha$-CifB antibodies were evaluated using an enzyme-linked immunosorbent assay, and titers were determined to be higher than 1:500,000 for each antibody. Using standard

protocols of the Invitrogen WesternDot kit, antibody specificity to $w \mathrm{Mel}+$ samples was verified using western blots (1:1000-fold antibody dilution) on protein isolated from homogenates of 50 testes pairs (0-8 hour old males) and 10 ovary pairs (6 day old females) from $w$ Mel+ (positive), $w$ Mel- (negative control), and $c i f A B$ transgenic (positive) flies. The correct size band was only

\section{$\underline{\text { NLS identification }}$}

CifA amino acid sequences from known Wolbachia and close relatives were input into the cNLS Mapper software [73] to identify putative NLS sequences within each protein (Table S3). cNLS Mapper identifies sequences specific to the importin $\alpha / \beta$ pathway. A cut-off score of 4 was applied to all sequences. Higher scores indicate stronger NLS activities. Scores $>8$ indicate exclusive localization to the nucleus, 7-8 indicate partial localization to the nucleus, 3-5 indicate localization to both the nucleus and the cytoplasm, and score 1-2 indicate localization exclusively to the cytoplasm. Predicted NLS sequences are divided into monopartite and bipartite classes. Monopartite NLSs contain a single region of basic residues, and bipartite NLSs contain two regions of basic residues separated by a linker region.

\section{Development of transgenic lines}

A cifA variant was synthesized de novo at GenScript and cloned into a pUC57 plasmid as described previously [48]. Site-directed mutagenesis was performed by GenScript to produce the 
mutants outlined in Figure 5. The cifA 189 variant was first described in Shropshire et al. [48] as cif $A_{2}$. UAS transgenic cifA mutant flies were then generated using previously established protocols [13]. Briefly, GenScript sub-cloned each gene into the pTIGER plasmid, a pUASpbased vector designed for germline-specific expression. Transgenes were then integrated into $\mathrm{y}^{1} \mathrm{M}\{$ vas-int.Dm $\} Z \mathrm{ZH}-2 \mathrm{~A} \mathrm{w}^{*} ; \mathrm{P}\{\mathrm{CaryP}\}$ attP40 attachment sites into the D. melanogaster genome using PhiC31 integrase via embryonic injections by BestGene. At least 200 embryos were injected per transgenic construct, and successful transformants were identified based on red eye color gene included on the pTIGER plasmid containing the transgene. All sequences are reported in Table S4.

\section{Fly rearing and strains}

D. melanogaster stocks $\mathrm{y}^{1} \mathrm{w}^{*}$ (BDSC 1495), nos-GAL4:VP16 (BDSC 4937), UAS transgenic lines homozygous for cifA, cifB, cifAB, WD0508 [13] and Protamine mutant (w[1118]; $\Delta \mathrm{Mst35B[floxed],} \mathrm{Sco/CyO)} \mathrm{[74]} \mathrm{were} \mathrm{maintained} \mathrm{on} \mathrm{a} \mathrm{12-hour} \mathrm{light/dark} \mathrm{cycle} \mathrm{at}$ $25^{\circ} \mathrm{C}$ and $70 \%$ relative humidity on $50 \mathrm{~mL}$ of standard media. Uninfected protamine mutant line was generated by three generations of tetracycline treatment $(20 \mu \mathrm{g} / \mathrm{ml}$ in $50 \mathrm{ml}$ of fly media) as described in previous studies [13], followed by two rounds of rearing on standard food media before using in the experiments. Infection status for all lines was regularly confirmed by PCR using Wolb_F and Wolb_R3 primers [75].

\section{$\underline{\text { Hatch rates }}$}

Parental flies were either wild type uninfected ( $w$ Mel-) or infected $(w \mathrm{Mel}+)$ with Wolbachia or transgene-expressing with no Wolbachia infection. Uninfected transgenic flies 
were generated previously [13,17]. Paternal grandmother age was controlled to 9-11 days for expression of naturally high penetrance of $w \mathrm{Mel}$ CI [76]. Parental transgenic males were generated through crossing nos-Gal4:VP16 virgin females (aged 9-11 days) to UAS-cif transgenic, uninfected males [76]. Mothers were aged 6-9 days before crossing, while father males first emerged between 0-8 hours were used in hatch rates and tissue collections to control for the younger brother effect associated with lower CI penetrance [13,77].

Hatch rates were set up as described previously [13,17]. Briefly, a male and female pair was placed in an 8oz, round bottom, polypropylene Drosophila stock bottle with a grape juiceagar plate containing a small amount of yeast placed at the base and secured with tape. These bottles were then placed in a $25^{\circ} \mathrm{C}$ incubator overnight to allow for courting and mating. The following day, these plates were discarded and replaced with new grape juice-agar plates with fresh yeast. After an additional 24 hours, the plates were removed, and the embryos were counted. The embryo plates were then incubated for 36 hours at $25^{\circ} \mathrm{C}$ before the total number of unhatched embryos were counted. Any crosses with fewer than 25 embryos laid were discarded from the analyses. Statistical significance $(\mathrm{p}<0.05)$ was determined by a Kruskal-Wallis test and Dunn's multiple test correction in GraphPad Prism 7. All p-values are listed in Table S2.

\section{Immunofluorescence: testes and seminal vesicles}

Siblings from the hatch rate (males 0-8 hours) were collected for testes dissection in icecold 1x PBS solution. Tissues were fixed in $4 \%$ formaldehyde diluted in 1x PBS for 30 min at room temperature and washed in 1x PBS-T $(1 \mathrm{x}$ PBS $+0.3 \%$ TritonX-100) three times for $5 \mathrm{~min}$ each. Tissues were then blocked in $1 \%$ BSA in PBS-T for 1 hour at room temperature. They were then incubated with $1^{\circ}$ antibody ( $\alpha$-CifA 1:500 OR $\alpha$-CifB 1:500) overnight at $4^{\circ} \mathrm{C}$ 
rotating. After washing in 1x PBS-T three times for 5 min each at room temperature, they were incubated with 1:1000 dilution Goat anti-rabbit Alexa Fluor 594 secondary antibody (Fisher Scientific, Cat\#A11037) for 4 hours at room temperature in the dark. Tissues were then washed three times for 5 minutes each in $1 \mathrm{X}$ PBS-T and mounted on slides. To stain the nuclear DNA, $0.2 \mathrm{mg} / \mathrm{mL}$ of DAPI was added to the mounting media before the coverslip was gently placed over the tissue and excess liquid wiped away. Slides were allowed to dry overnight in the dark before viewing on the Zeiss LSM 880 confocal microscope. All images were acquired with the 402

\section{Decondensation of Mature Sperm Nuclei}

Squashed seminal vesicles collected from male flies (aged 0-8 hours) were treated with $10 \mathrm{mM}$ DTT, $0.2 \%$ Triton X-100, and $400 \mathrm{U}$ heparin in $1 \mathrm{X}$ PBS for $30 \mathrm{~min}$ [34]. The slides were then washed quickly in 1X PBS before immunofluorescence staining (see above).

\section{Immunofluorescence and quantification: Histones}

Testes from male flies (aged 0-8 hours) were fixed and stained as described above for testes. The tissues were stained with a core histone antibody (Millipore Sigma, Cat\#MABE71) canoe stage bundles containing histones relative to total bundles from each individual testis were graphed in GraphPad Prism 7. Statistical significance $(\mathrm{p}<0.05)$ were determined by pairwise comparisons based on Kolmogorov-Smirnov test and multiple comparisons based on a KruskalWallis test and Dunn's multiple test correction in GraphPad Prism 7. 
$418 \quad$ Sperm isolation and chromomycin A3 staining/quantification

419 Seminal vesicles were collected from male flies (aged 0-8 hours for 1-day old flies and 7420 days for older flies) and placed on a microscope slide in ice-cold 1x PBS. Sperm was extracted 421 on the slide using forceps and fixed in 3:1 vol/vol methanol:acetic acid at $4{ }^{\circ} \mathrm{C}$ for 20 min. Excess 422 solution was then removed, and the slide was air dried. Each slide was treated in the dark for 20 423 min with $0.25 \mathrm{mg} / \mathrm{mL}$ of CMA3 in McIlvain's buffer, $\mathrm{pH} 7.0$, with $10 \mathrm{mM} \mathrm{MgCl2}$. Sperm was 424 then washed in 1x PBS, mounted, and imaged using a Keyence BZ-X700 Fluorescence 425 microscope. All images were acquired with the same parameters for each line and did not 426 undergo significant alteration. Fluorescence quantification was performed by scoring fluorescent 427 pixels in arbitrary units (A.U.) within individual sperm heads using ImageJ as per the details 428 described in [78], and calculated fluorescence intensity per sperm head was graphed. Statistical 429 significance $(\mathrm{p}<0.05)$ was determined by a Kruskal-Wallis test and Dunn's multiple test 430 correction in GraphPad Prism 7. All of the experiments involving CMA3 staining were 431 performed at $21^{\circ} \mathrm{C}$ instead of $25^{\circ} \mathrm{C}$. CI hatch rate assays were run in parallel to ensure that CI and 432 rescue phenotypes are not impacted due to changed temperature conditions.

\section{Immunofluorescence: ovaries}

435 Ovaries from females (6 days old) were dissected in 1x PBS on ice and processed as 436 described previously [77,79]. Tissues were blocked in 1\% BSA in PBS-T for 1 hour at room 437 temperature and were first incubated with $\alpha$-CifA $(1: 500)$ primary antibody at $4^{0} \mathrm{C}$ overnight. 438 After washing in 1x PBS-T three times for 5 min each at room temperature, they were incubated 439 with 1:1000 dilution Alexa Fluor 488 secondary antibody (Thermo Fisher Scientific, 
440 Cat\#A11034) for 4 hours at room temperature in the dark. Samples were then rinsed properly $441 \quad$ and blocked again before incubating with $\alpha$-ftsZ (1:150) primary antibody (a kind gift from Dr. 442 Irene Newton) to stain Wolbachia at $4^{0} \mathrm{C}$ overnight. After washing in $1 \mathrm{x}$ PBS-T three times, 443 samples were incubated with second secondary antibody (Alexa Fluor 594) for 4 hours in the 444 dark. Since both CifA and ftsZ antibodies were generated in the same animal, we used secondary 445 antibodies conjugated to two distant fluorophores to distinguish specific signals. Tissues were 446 then washed three times for 5 minutes each in $1 \mathrm{X}$ PBS, stained with DAPI to label nuclear DNA 447 and mounted on slides. Slides were allowed to dry overnight in the dark before viewing on the $448 \quad$ Zeiss LSM 880 confocal microscope.

\section{Immunofluorescence: embryos}

After 24 hours of mating, plates were switched, and embryos were collected every 30 minutes. Embryos were collected in a $100 \mu \mathrm{m}$ mesh basket in embryo wash solution. To remove the chorion, the basket was placed in $50 \%$ bleach for 3 min and then rinsed with $1 \mathrm{x}$ PBS. The embryos were then transferred to 50:50 4\% paraformaldehyde (PFA) and heptane in a microcentrifuge tube and rotated for $20 \mathrm{~min}$ at room temperature. Tubes were then removed from the rotator, and the heptane and PFA were allowed to separate before the bottom PFA phase was new volume of methanol was added to the embryos, and they were allowed to settle to the 
bioRxiv preprint doi: https://doi.org/10.1101/2022.01.15.476471; this version posted February 23, 2022. The copyright holder for this preprint (which was not certified by peer review) is the author/funder, who has granted bioRxiv a license to display the preprint in perpetuity. It is made available under aCC-BY-NC-ND 4.0 International license. 
Acknowledgments: The authors thank Jennifer Battle for her assistance in fly collections and staining for sperm integrity assays, Alex Mansueto for his assistance in hatch rates, and Sarah Bordenstein, Dylan Shropshire, and Luis Mendez for providing helpful feedback on the manuscript. We thank Dr. Janna McLean for sending the Protamine mutant fly line for conducting the experiments. We thank Dr. Irene Newton for sharing Wolbachia antibody and providing useful feedback on the preprint version of this manuscript. We also thank the Cell

Funding: Digestive Disease Research Center Scholarships S1848284, S1848300, S1883559,

Author contributions: Conceptualization, B.A.L. and S.R.B.; Methodology, B.A.L., R.K. and DK020593 to CISR.

Competing interests: Authors declare no competing interests. 
bioRxiv preprint doi: https://doi.org/10.1101/2022.01.15.476471; this version posted February 23, 2022. The copyright holder for this preprint

(which was not certified by peer review) is the author/funder, who has granted bioRxiv a license to display the preprint in perpetuity. It is made available under aCC-BY-NC-ND 4.0 International license.

485 Data and materials availability: All data are available in the main text or the supplementary

486 materials. Unique biological materials will be available upon request. 


\section{References:}

1. Bordenstein SR, Ohara FP, Werren JH. Wolbachia-induced incompatibility precedes other hybrid incompatibilities in Nasonia. Nature. 2001;409: 707-710. doi:10.1038/35055543

2. Charlat S, Hurst GDD, Merçot H. Evolutionary consequences of Wolbachia infections. Trends Genet. 2003;19: 217-223. doi:10.1016/S0168-9525(03)00024-6 infection in Drosophila. PLoS Biol. 2006;4: 1852-1862. doi:10.1371/journal.pbio.0040325

4. Bian G, Xu Y, Lu P, Xie Y, Xi Z. The Endosymbiotic Bacterium Wolbachia Induces Resistance to Dengue Virus in Aedes aegypti. PLoS Pathog. 2010;6: e1000833. doi:10.1371/journal.ppat.1000833

5. Frentiu FD, Zakir T, Walker T, Popovici J, Pyke AT, van den Hurk A, et al. Limited Wolbachia. PLoS Negl Trop Dis. 2014;8. doi:10.1371/journal.pntd.0002688

6. Walker T, Johnson PH, Moreira LA, Iturbe-Ormaetxe I, Frentiu FD, McMeniman CJ, et populations. Nature. 2011;476: 450-455. doi:10.1038/nature10355

7. Bourtzis K, Dobson SL, Xi Z, Rasgon JL, Calvitti M, Moreira LA, et al. Harnessing mosquito-Wolbachia symbiosis for vector and disease control. Acta Trop. 2014;132. doi:10.1016/j.actatropica.2013.11.004

8. Hughes GL, Koga R, Xue P, Fukatsu T, Rasgon JL. Wolbachia infections are virulent and inhibit the human malaria parasite Plasmodium falciparum in Anopheles gambiae. PLoS Pathog. 2011;7: e1002043. doi:10.1371/journal.ppat.1002043 
9. O’Connor L, Plichart C, Sang AC, Brelsfoard CL, Bossin HC, Dobson SL. Open release of male mosquitoes infected with a wolbachia biopesticide: field performance and infection containment. PLoS Negl Trop Dis. 2012;6: e1797. doi:10.1371/journal.pntd.0001797

10. Taylor MJ, Bordenstein SR, Slatko B. Microbe profile: Wolbachia: A sex selector, a viral protector and a target to treat filarial nematodes. Microbiol (United Kingdom). 2018. doi:10.1099/mic.0.000724

11. Shropshire JD, Leigh B, Bordenstein SR, Initiative M. Symbiont-mediated cytoplasmic incompatibility $\square$ : What have we learned in 50 years? Elife. 2020; 1-51. doi:10.20944/preprints202008.0350.v1

12. Turelli M. Evolution of incompatibility-inducing microbes and their hosts. Evolution (N Y). 1994;48: 1500-1513. doi:10.1111/j.1558-5646.1994.tb02192.x

13. LePage DP, Metcalf JA, Bordenstein SR, On J, Perlmutter JI, Shropshire JD, et al. Prophage WO genes recapitulate and enhance Wolbachia-induced cytoplasmic incompatibility. Nature. 2017;543: 243-247. doi:10.1038/nature21391

14. Bordenstein SR, Bordenstein SR. Eukaryotic association module in phage WO genomes from Wolbachia. Nat Commun. 2016;7. doi:10.1038/ncomms13155

15. Beckmann JF, Ronau JA, Hochstrasser M. A Wolbachia deubiquitylating enzyme induces cytoplasmic incompatibility. Nat Microbiol. 2017;2. doi:10.1038/nmicrobiol.2017.7

16. Shropshire JD, Bordenstein SR. Two-by-one model of cytoplasmic incompatibility: Synthetic recapitulation by transgenic expression of cifa and cifb in drosophila. PLoS Genet. 2019;15. doi:10.1371/journal.pgen.1008221

17. Shropshire JD, On J, Layton EM, Zhou H, Bordenstein SR. One prophage WO gene 
rescues cytoplasmic incompatibility in Drosophila melanogaster. Proc Natl Acad Sci U S

536
A. 2018;115: 4987-4991. doi:10.1073/pnas.1800650115

18. Adams KL, Abernathy DG, Willett BC, Selland EK. Wolbachia cifB induces cytoplasmic incompatibility in the malaria mosquito. 2021. doi:10.1101/2021.04.20.440637

19. Sun G, Zhang M, Chen H, Hochstrasser M. The Wolbachia CinB Nuclease is Sufficient for Induction of Cytoplasmic Incompatibility. bioRxiv. 2021; 2021.10.22.465375. Available: https://www.biorxiv.org/content/10.1101/2021.10.22.465375v1\%0Ahttps://www.biorxiv.o rg/content/10.1101/2021.10.22.465375v1.abstract

20. Yang P, Wu W, Macfarlan TS. Maternal histone variants and their chaperones promote paternal genome activation and boost somatic cell reprogramming. BioEssays. 2015;37: 52-59. doi:10.1002/bies.201400072

21. Landmann F, Orsi GA, Loppin B, Sullivan W. Wolbachia-mediated cytoplasmic incompatibility is associated with impaired histone deposition in the male pronucleus. PLoS Pathog. 2009;5. doi:10.1371/journal.ppat.1000343

22. Lassy CW, Karr TL. Cytological analysis of fertilization and early embryonic development in incompatible crosses of Drosophila simulans. Mech Dev. 1996;57: 47-58. doi:10.1016/0925-4773(96)00527-8

23. Serbus LR, Casper-Lindley C, Landmann F, Sullivan W. The genetics and cell biology of Wolbachia-host interactions. Annu Rev Genet. 2008;42: 683-707. doi:10.1146/annurev.genet.41.110306.130354

24. Tram U, Sullivan W. Role of delayed nuclear envelope breakdown and mitosis in Wolbachia-induced cytoplasmic incompatibility. Science (80- ). 2002;296: 1124-1126. 
doi:10.1126/science. 1070536

25. Tram U, Fredrick K, Werren JH, Sullivan W. Paternal chromosome segregation during the first mitotic division determines Wolbachia-induced cytoplasmic incompatibility phenotype. J Cell Sci. 2006;119: 3655-3663. doi:10.1242/jcs.03095

26. Callaini G, Riparbelli MG, Giordano R, Dallai R. Mitotic defects associated with cytoplasmic incompatibility in Drosophila simulans. J Invertebr Pathol. 1996;67: 55-64. doi:10.1006/jipa.1996.0009

27. Callaini G, Dallai R, Riparbelli MG. Wolbachia-induced delay of paternal chromatin condensation does not prevent maternal chromosomes from entering anaphase in incompatible crosses of Drosophila simulans. J Cell Sci. 1997;110 ( Pt 2: 271-280. Toxin-Antidote Model of Cytoplasmic Incompatibility: Genetics and Evolutionary Implications. Trends in Genetics. 2019. pp. 175-185. doi:10.1016/j.tig.2018.12.004

29. Shropshire JD, Leigh B, Bordenstein SR, Duplouy A, Riegler M, Brownlie JC, et al. Models and Nomenclature for Cytoplasmic Incompatibility: Caution over Premature Conclusions - A Response to Beckmann et al. Trends in Genetics. 2019. pp. 397-399. doi:10.1016/j.tig.2019.03.004

30. Horard B, Terretaz K, Gosselin-Grenet A-S, Sobry H, Sicard M, Landmann F, et al.

31. Fuller M. The Development of Drosophila melanogaster. Cold Spring Harbor Laboratory 
Protamines and Mst77F of Histones during Chromatin Condensation in Late Spermatids and Role of Sesame in the Removal of These Proteins from the Male Pronucleus . Mol Cell Biol. 2005;25: 6165-6177. doi:10.1128/mcb.25.14.6165-6177.2005

33. Fabian L, Brill JA. Drosophila spermiogenesis. Spermatogenesis. 2012;2: 197-212. doi:10.4161/spmg.21798

34. Eren-Ghiani Z, Rathke C, Theofel I, Renkawitz-Pohl R. Prt199C Acts Together with Protamines and Safeguards Male Fertility in Drosophila. Cell Rep. 2015;13: 2327-2335. doi:10.1016/j.celrep.2015.11.023

35. Bonnefoy E, Orsi GA, Couble P, Loppin B. The essential role of Drosophila HIRA for de novo assembly of paternal chromatin at fertilization. PLoS Genet. 2007;3: 1991-2006. doi:10.1371/journal.pgen.0030182

36. Bressac C, Rousset F. The reproductive incompatibility system in Drosophila simulans: Dapi-staining analysis of the Wolbachia symbionts in sperm cysts. J Invertebr Pathol. 1993;61: 226-230. doi:10.1006/jipa.1993.1044

37. Clark ME, Veneti Z, Bourtzis K, Karr TL. The distribution and proliferation of the intracellular bacteria Wolbachia during spermatogenesis in Drosophila. Mech Dev. 2002;111: 3-15. Available: http://www.ncbi.nlm.nih.gov/pubmed/11804774 configuration during spermiogenesis in Drosophila. J Cell Sci. 2007;120: 1689-1700. doi:10.1242/jcs.004663

39. Brunner AM, Nanni P, Mansuy IM. Epigenetic marking of sperm by post-translational modification of histones and protamines. Epigenetics and Chromatin. 2014;7. 
604

605

606

607

608

609

610

611

612

613

614

615

616

617

618

doi:10.1186/1756-8935-7-2

40. Luense LJ, Donahue G, Lin-Shiao E, Rangel R, Weller AH, Bartolomei MS, et al. Gcn5Mediated Histone Acetylation Governs Nucleosome Dynamics in Spermiogenesis. Dev Cell. 2019;51: 745-758.e6. doi:10.1016/j.devcel.2019.10.024

41. Goudarzi A, Shiota H, Rousseaux S, Khochbin S. Genome-scale acetylation-dependent histone eviction during spermatogenesis. J Mol Biol. 2014;426: 3342-3349. doi:10.1016/j.jmb.2014.02.023

42. Fenic I, Sonnack V, Failing K, Bergmann M, Steger K. In vivo effects of histonedeacetylase inhibitor trichostatin-A on murine spermatogenesis. J Androl. 2004;25: 811818. doi:10.1002/j.1939-4640.2004.tb02859.x

43. Sonnack V, Failing K, Bergmann M, Steger K. Expression of hyperacetylated histone H4 during normal and impaired human spermatogenesis. Andrologia. 2002;34: 384-390. doi:10.1046/j.1439-0272.2002.00524.x

44. Lolis D, Georgiou I, Syrrou M, Zikopoulos K, Konstantelli M, Messinis I. Chromomycin A3-staining as an indicator of protamine deficiency and fertilization. Int J Androl. 1996;19: 23-27. doi:10.1111/j.1365-2605.1996.tb00429.x

45. Kazerooni T, Asadi N, Jadid L, Kazerooni M, Ghanadi A, Ghaffarpasand F, et al. Evaluation of sperm's chromatin quality with acridine orange test, chromomycin A3 and aniline blue staining in couples with unexplained recurrent abortion. J Assist Reprod Genet. 2009;26: 591-596. doi:10.1007/s10815-009-9361-3

46. Kosugi S, Hasebe M, Tomita M, Yanagawa H. Systematic identification of cell cycledependent yeast nucleocytoplasmic shuttling proteins by prediction of composite motifs. Proc Natl Acad Sci U S A. 2009;106: 10171-10176. doi:10.1073/pnas.0900604106 
47. $\quad$ Lindsey ARI, Rice DW, Bordenstein SR, Brooks AW, Bordenstein SR, Newton ILG.

Evolutionary Genetics of Cytoplasmic Incompatibility Genes cifA and cifB in Prophage WO of Wolbachia. Genome Biol Evol. 2018;10: 434-451. doi:10.1093/gbe/evy012

48. Shropshire JD, Kalra M, Bordenstein SR. Evolution-guided mutagenesis of the cytoplasmic incompatibility proteins: Identifying CifA's complex functional repertoire and new essential regions in CifB. PLoS Pathog. 2020;16: e1008794. doi:10.1371/journal.ppat.1008794

49. Kosugi S, Hasebe M, Matsumura N, Takashima H, Miyamoto-Sato E, Tomita M, et al. Six classes of nuclear localization signals specific to different binding grooves of

50. Ferree PM, Frydman HM, Li JM, Cao J, Wieschaus E, Sullivan W. Wolbachia utilizes host microtubules and Dynein for anterior localization in the Drosophila oocyte. PLoS Pathog. 2005;1: e14. doi:10.1371/journal.ppat.0010014

51. Marlow FL. Maternal Control of Development in Vertebrates. Colloq Ser Dev Biol. cytoplasmic incompatibility enzyme CIDB targets nuclear import and protamine-histone exchange factors. Elife. 2019;8. doi:10.7554/eLife.50026

53. Braun RE. Packaging paternal chromosomes with protamine. Nat Genet. 2001;28: 10-12. doi:10.1038/ng0501-10

54. Sassone-Corsi P. Editorial: Never enough - On the multiplicity and uniqueness of transcriptional regulators in postmeiotic male germ cells. Endocrinology. 2002;143: 15751577. doi:10.1210/endo.143.5.8874 
55. Gaucher J, Reynoird N, Montellier E, Boussouar F, Rousseaux S, Khochbin S. From meiosis to postmeiotic events: The secrets of histone disappearance. FEBS J. 2010;277: 599-604. doi:10.1111/j.1742-4658.2009.07504.x

56. Rathke C, Baarends WM, Awe S, Renkawitz-Pohl R. Chromatin dynamics during spermiogenesis. Biochim Biophys Acta - Gene Regul Mech. 2014;1839: 155-168. doi:10.1016/j.bbagrm.2013.08.004

57. Schagdarsurengin U, Paradowska A, Steger K. Analysing the sperm epigenome: Roles in early embryogenesis and assisted reproduction. Nat Rev Urol. 2012;9: 609-619. doi:10.1038/nrurol.2012.183

58. Carrell DT, Hammoud SS. The human sperm epigenome and its potential role in embryonic development. Mol Hum Reprod. 2009;16: 37-47. doi:10.1093/molehr/gap090

59. Kaneshiro KR, Rechtsteiner A, Strome S. Sperm-inherited H3K27me3 impacts offspring transcription and development in C. elegans. Nat Commun. 2019;10. doi:10.1038/s41467019-09141-w

60. Levine MT, Vander Wende HM, Malik HS. Mitotic fidelity requires transgenerational action of a testis-restricted HP1. Elife. 2015;4: 1-20. doi:10.7554/eLife.07378

61. Hundertmark T, Gärtner SMK, Rathke C, Renkawitz-Pohl R. Nejire/dCBP-mediated histone $\mathrm{H} 3$ acetylation during spermatogenesis is essential for male fertility in Drosophila melanogaster. PLoS One. 2018;13. doi:10.1371/journal.pone.0203622

62. Aoki VW, Emery BR, Liu L, Carrell DT. Protamine levels vary between individual sperm cells of infertile human males and correlate with viability and DNA integrity. J Androl. 2006;27: 890-898. doi:10.2164/jandrol.106.000703

63. Cho C, Jung-Ha H, Willis WD, Goulding EH, Stein P, Xu Z, et al. Protamine 2 deficiency 
673

674

675

676

677

678

679

680

681

682

683

684

685

686

687

688

689

690

691

692

693

694

695

leads to sperm DNA damage and embryo death in mice. Biol Reprod. 2003;69: 211-217. doi:10.1095/biolreprod.102.015115

64. Loppin B, Lepetit D, Dorus S, Couble P, Karr TL. Origin and neofunctionalization of a Drosophila paternal effect gene essential for zygote viability. Curr Biol. 2005;15: 87-93. doi:10.1016/j.cub.2004.12.071

65. Clark ME, Heath BD, Anderson CL, Karr TL. Induced paternal effects mimic cytoplasmic incompatibility in Drosophila. Genetics. 2006;173: 727-734.

doi:10.1534/genetics.105.052431

66. Karr TL. Intracellular sperm/egg interactions in Drosophila: A three-dimensional structural analysis of a paternal product in the developing egg. Mech Dev. 1991;34: 101111. doi:10.1016/0925-4773(91)90047-A

67. Evans JP. The molecular basis of sperm-oocyte membrane interactions during mammalian fertilization. Hum Reprod Update. 2002;8: 297-311. doi:10.1093/humupd/8.4.297

68. Flesch FM, Gadella BM. Dynamics of the mammalian sperm plasma membrane in the process of fertilization. Biochim Biophys Acta - Rev Biomembr. 2000;1469: 197-235. doi:10.1016/S0304-4157(00)00018-6

69. Degrugillier ME, Leopold RA. Ultrastructure of sperm penetration of house fly eggs. J Ultrasructure Res. 1976;56: 312-325. doi:10.1016/S0022-5320(76)90006-X

70. Xiao Y, Chen H, Wang H, Zhang M, Chen X, Berk JM, et al. Structural and mechanistic insights into the complexes formed by Wolbachia cytoplasmic incompatibility factors . Proc Natl Acad Sci. 2021;118: e2107699118. doi:10.1073/pnas.2107699118

71. McLay DW, Clarke HJ. Remodelling the paternal chromatin at fertilization in mammals. Reproduction. 2003;125: 625-633. doi:10.1530/rep.0.1250625 
696 72. Landmann F, Orsi GA, Loppin B, Sullivan W. Wolbachia-mediated cytoplasmic incompatibility is associated with impaired histone deposition in the male pronucleus. PLoS Pathog. 2009;5. doi:10.1371/journal.ppat.1000343

73. Zhang B, Ye W, Ye Y, Zhou H, Saeed AFUH, Chen J, et al. Structural insights into Cas13b-guided CRISPR RNA maturation and recognition. Cell Res. 2018;28: 1198-1201. doi:10.1038/s41422-018-0109-4

74. Tirmarche S, Kimura S, Sapey-Triomphe L, Sullivan W, Landmann F, Loppin B. morphology but are dispensable for male fertility. G3 Genes, Genomes, Genet. 2014;4: 2241-2245. doi:10.1534/g3.114.012724

75. Casiraghi M, Bordenstein SR, Baldo L, Lo N, Beninati T, Wernegreen JJ, et al. Phylogeny of Wolbachia pipientis based on gltA, groEL and ftsZ gene sequences $\square$ : clustering of arthropod and nematode symbionts in the F supergroup, and evidence for further diversity in the Wolbachia tree. 2006; 4015-4022. doi:10.1099/mic.0.28313-0 melanogaster. MBio. 2019;10. doi:10.1128/mBio.01879-19

77. Shropshire JD, Hamant E, Cooper BS. Male Age and Wolbachia Dynamics: Investigating How Fast and Why Bacterial Densities and Cytoplasmic Incompatibility Strengths Vary. MBio. 2021;12. doi:10.1128/mbio.02998-21 
bioRxiv preprint doi: https://doi.org/10.1101/2022 01.15.476471; this version posted February 23,2022 . The copyright holder for this preprint

(which was not certified by peer review) is the author/funder, who has granted bioRxiv a license to display the preprint in perpetuity. It is made available under aCC-BY-NC-ND 4.0 International license.

719 79. Newton ILG, Savytskyy O, Sheehan KB. Wolbachia Utilize Host Actin for Efficient Maternal Transmission in Drosophila melanogaster. PLoS Pathog. 2015;11. doi:10.1371/journal.ppat.1004798 
Figure legends

\section{Fig 1. CifA and CifB invade sperm nuclei during spermatogenesis and spermiogenesis.} Schematic representation of Drosophila melanogaster male reproductive system created by Biorender is shown on the top. Testes $(n=20)$ from $<8$ hrs old males expressing dual transgenes cif $A B$ were dissected and immunostained to visualize CifA (green) and CifB (red) during sperm morphogenesis. DAPI stain (blue) was used to label nuclei. CifA, but not CifB, localizes in the germline stem cells at the apical end of testes. Both CifA and CifB localize in the nuclei of mitotic spermatogonium, spermatocytes, and round onion stage spermatids. In the later stages of spermiogenesis, elongating spermatids harbor CifA and CifB at the acrosomal tip of the heads. CifB is present in all canoe-stage spermatid nuclei, whereas CifA is present on average in $39 \%$ of spermatids per bundle. Cifs are not accessible by the antibodies in the tightly compacted spermatids at the needle-stage. After decondensing mature sperms isolated from seminal vesicles (see methods), CifA and CifB are detectable in the acrosome regions at varying percentages. CifA is common among sperm tails in a speckled pattern (white arrow) and either present on average in $45 \%$ or $0 \%$ of the mature sperm heads depending upon the sampled seminal vesicles. CifA's presence in the acrosome region is shown by solid white arrowheads and absence with empty white arrowheads. CifB is present in acrosomal tips of all of the sperms (solid white arrowheads) and does not occur with sperm tails. CifA and CifB localization patterns are similar in wild type $(w \mathrm{Mel}+)$ line and signals are absent in Wolbachia-uninfected (wMel-) negative control line (Fig S2). Some of the CifA and CifB proteins are also stripped by the individualization complex into the cytoplasmic waste bag (Fig S5). The experiment was repeated in two biological replicates.

Fig 2. Cifs cause histone retention in late canoe spermatids and protamine deficiency in mature sperms. (A) Testes $(\mathrm{n}=15)$ from $<8 \mathrm{hrs}$ old males of $w \mathrm{Mel}+, w \mathrm{Mel}$ - and transgenic $c$ if $A B$ lines were dissected and immunostained to visualize and quantify spermatid bundles with histone retention (purple) during late canoe stage of spermatogenesis. DAPI stain (blue) was used to label spermatid nuclei. Total spermatid bundles with DAPI signals and those with retained Histones were manually counted and graphed. Compared to the negative control $w \mathrm{Mel}-, w \mathrm{Mel}+$ Wolbachia and dually-expressed $c i f A B$ transgenic lines show abnormal histone retention in the late canoe stage. Vertical bars represent mean, and error bars represent standard deviation. Letters indicate statistically significant $(\mathrm{p}<0.05)$ differences as determined by pairwise comparisons based on Kolmogorov-Smirnov test. (B) Mature sperms isolated from seminal vesicles $(n=15)$ of $<8 \mathrm{hr}$ old males reared at $21^{\circ} \mathrm{C}$ were stained with fluorescent CMA3 (green) for detection of protamine deficiency in each individual sperm nucleus. Individual sperm head intensity was quantified in ImageJ (see methods) and graphed. $w \mathrm{Mel}+$ and transgenic cifAB lines show enhanced protamine deficiency levels compared to $w \mathrm{Mel}$ - control. Vertical bars represent mean and error bars represent standard deviation. Letters indicate statistically significant $(\mathrm{p}<0.05)$ differences as determined by multiple comparisons based on a Kruskal-Wallis test and 
Dunn's multiple test correction. All of the P-values are reported in Table S1. The experiments were performed in two independent biological replicates and samples were blind-coded for the first run. Raw data underlying this figure can be found in $\mathrm{S} 1$ data file.

Fig 3. Protamine mutants enhance wild type CI and show significantly increased levels of protamine deficiency in mature sperms. (A) Sperms from the Wolbachia-infected $(\Delta \operatorname{Prot}+)$ and -uninfected ( $\triangle$ Prot-) protamine mutant (w[1118]; $\Delta$ Mst35B[floxed], Sco/CyO) males exhibit significantly increased CMA3 fluorescence indicative of protamine deficiency compared to both wild type $w \mathrm{Mel}+$ and $w \mathrm{Mel}-$. Vertical bars represent mean, and error bars represent standard deviation. Letters indicate statistically significant $(\mathrm{p}<0.05)$ differences as determined by multiple comparisons based on a Kruskal-Wallis test and Dunn's multiple test correction. All of the Pvalues are reported in Table S1. (B) CI hatch rate analyses of male siblings used in CMA3 assays (panel A) validate that $\Delta$ Prot+ males with increased sperm protamine deficiency causes stronger (rescuable) CI levels than $w \mathrm{Mel}+. \Delta$ Prot- males do not cause CI. Letters to the right indicate statistically significant $(\mathrm{p}<0.05)$ differences as determined by pairwise Mann-Whitney test and multiple comparisons calculated using a Kruskal-Wallis test and Dunn's multiple test correction. All of the P-values related to CI assay are reported in Table S2. Raw data underlying this figure can be found in $\mathrm{S} 1$ data file. levels. (A) Schematic representation of CifA annotation shows the annotated bipartite nuclear localization signal (bNLS) with engineered amino acid substitutions and deletions. (B, C) Hatch rate assays assessed both $\mathrm{CI}(\mathrm{B})$ and rescue $(\mathrm{C})$ in flies expressing wild type, transgenic, and mutant cifA. Each dot represents the percent of embryos that hatched from a single male and female pair. Sample size is listed in parentheses. Horizontal bars represent the median. Letters to the right indicate significant differences determined by a Kruskal-Wallis test and Dunn's multiple comparison tests. All the P-values are reported in Table S2. (D) Antibody labeling (green) and DAPI staining of onion stage spermatids in the testes of the bNLS mutant line $\left(c i f A_{\triangle b N L S}\right)$ reveals that the deletion ablates CifA's localization to the nucleus, and CifA thus remains in the surrounding cytoplasm. The imaging experiment was conducted in parallel to nos; $c i f A B$ line shown in Fig 1. (E) Mature sperms isolated from seminal vesicles $(\mathrm{n}=15)$ of $<8 \mathrm{hr}$ old males of transgenic $c i f A_{\triangle b n l s} B$ line shows reduced fluorescence indicative of less Protamine deficiency compared to cifAB. To control for any background confounding effects of nosGal4VP16 driver line, wMel- fathers were prior crossed to nos- mothers to generate males with nos; wMel-genotype. CMA3 fluorescence levels of sperms isolated from nos; wMel-males were similar to $w \mathrm{Mel}$ - wild type lines used in previous assays in this study. Vertical bars represent mean and error bars represent standard deviation. Letters indicate statistically significant $(\mathrm{p}<0.05)$ differences as determined by multiple comparisons based on a Kruskal-Wallis test and Dunn's multiple test correction. All of the P-values are reported in Table S1 and raw data underlying this figure can be found in S1 data file. 

female reproductive system. Mature oocytes leave the ovary (OV) and reach the uterus (UT), where they can be fertilized prior to being laid. Sperms from males are stored in specialized organs - spermathecae (SP) and seminal receptacle (SR) shown in the box, which open into the UT for fertilization to occur. Schematic is created with BioRender (B) Transgenic cifABexpressing and $w$ Mel- males were crossed to $w$ Mel- females. 4 hrs post-fertilization, sperms isolated from females were decondensed and immunostained for localizing CifA (green) and CifB (red). DAPI stain (blue) was used to label nuclei. CifA is absent in sperm heads (empty arrowheads) and puctae are seen along the sperm tails (arrows). CifB is present in apical acrosomal tip of all of the sperm heads (solid arrowheads), with more distant signal in the more decondensed sperm nuclei. No Cifs are present in the sperms transferred from $w$ Mel- negative control males. (C) Individual sperm intensity quantification shows that protamine deficiency of sperms from $w \mathrm{Mel}+$ and transgenic $c i f A B$ males persists after transfer in the females compared to $w$ Mel- males. Sperm protamine deficiency from transgenic cifAB males also persists in the reproductive tract of $w \mathrm{Mel}+$ females. Vertical bars represent mean, and error bars represent standard deviation. Letters indicate statistically significant $(\mathrm{p}<0.05)$ differences as determined by multiple comparisons based on a Kruskal-Wallis test and Dunn's multiple test correction. All of the P-values are reported in Table S1 and the raw data underlying this panel can be found in S1 data file. (D) Representative images of CMA3-stained mature sperms (arrows) transferred from $w \mathrm{Mel}-, w \mathrm{Mel}+$ and transgenic $c i f A B$ males in $w \mathrm{Mel}$ - and $w \mathrm{Mel}+$ female reproductive systems are shown.

\section{Fig 6. CifA is present in early oogenesis and absent in late-stage egg chambers. Both CifA} and CifB are absent in CI and rescue embryos. (A) Schematic representation of Drosophila melanogaster ovariole at the top illustrates the stages of oogenesis from left to right. Image was created with BioRender. Immunostaining assay indicates localization of CifA (green) to the cyst DNA (blue labeled with DAPI) in region 1 of the germarium of Wolbachia-uninfected transgenic cifA line. In $w \mathrm{Mel}+$ line, CifA colocalizes with Wolbachia (red) in the germarium, nurse cells and oocyte cytoplasm along 2-8 stages of egg chambers. CifA is absent in stage 10 egg chamber, whereas Wolbachia signals persist. In the transgenic cifA line, we note the observed autofluorescence in green channel outlining the tissue morphology does not signify CifA signals. Ovariole images were manually adjusted in Affinity designer software to align egg chamber stages in the same plane. (B) Immunofluorescence of CifA (green) and CifB (red) in $\sim 30-60$ min old embryos obtained from rescue (cifAB male $\mathrm{x} w \mathrm{Mel}+$ female) and CI crosses (nos; cif $A B$ male $\mathrm{x} w$ Mel- female). Histone antibody labeling core-histones (magenta) was used as a positive control. Histone signals were detected colocalizing with host DNA, labelled with DAPI (blue), whereas no CifA and CifB signals were detected. Dotted white embryonic periphery is drawn 
around the embryo shape. White arrows indicate dividing nuclei post fertilization and arrowheads indicate polar bodies.

\section{Supplementary figure legends.}

Fig S1. Western blots using Cif antibodies reveal proteins at the proper size. Western blots were run on protein extracted from ovaries $(\mathrm{n}=10)$ of wild type infected $w \mathrm{Mel}+$, uninfected $w$ Mel-, cifA transgenic (cifA), and dual cifA;B expressing transgenic lines. The expected size for CifA is $\sim 54 \mathrm{kD}$. Western blots were run using anti-CifB antibody on testes $(\mathrm{n}=15)$ of wild-type infected (+), uninfected (-), and cifA;B transgenic (A;B) lines. Expected CifB size is $\sim 133 \mathrm{kD}$. Cifs are absent in $w \mathrm{Mel}$ - control and present at accurate size in $w \mathrm{Mel}+$ and cif expressing lines.

Fig S2. Cifs invade spermatid nuclei in wild type $\boldsymbol{w}$ Mel+ testes. Testes $(n=20)$ from $<8$ hrs old males of wild type $w \mathrm{Mel}+$ and $w \mathrm{Mel}$ - lines were dissected and immunostained to visualize CifA (green) and CifB (red) during sperm morphogenesis. DAPI stain (blue) was used to label nuclei. CifA and CifB localization patterns in wild type lines are similar to that of transgenic cifAB (Fig 1) and signals are absent in $w$ Mel- uninfected control line. The experiment was conducted in parallel to the one shown in Fig 1.

Fig S3. CifA and CifB vary in abundance levels in spermatids and mature sperms. (A) ImageJ-based signal intensity quantification indicates CifA (green) is more abundantly expressed than CifB (red) in the spermatogonium stage of the spermatogenesis. Mean of individual data points with standard deviation is plotted on the graph. Letters indicate statistically significant $(\mathrm{p}<0.05)$ differences as determined by pairwise comparisons based on a Mann-Whitney test. Pvalues are reported in Table S1. (B) In the decondensed mature sperms isolated from seminal vesicles, CifB is present in the acrosomal tip of canoe-shaped spermatids and mature sperm heads, whereas CifA is present in only $40 \%$ and $20 \%$ of them, respectively. Quantification was performed on the images obtained in Fig 1 data. Each dot represents percentage of Cifs present in spermatids or mature sperms per testes examined. Raw data underlying this figure can be found in S1 data file.

Fig S4. Cif A and CifB are not detectable in the condensed mature sperms in seminal vesicles due to technical limitations. Seminal vesicles $(n=20)$ from $<8$ hrs old males of transgenic $c i f A B$, wild type $w \mathrm{Mel}+$ and $w \mathrm{Mel}-$ lines were dissected and immunostained to visualize CifA (green) and CifB (red) in the mature condensed sperms (indicated by white arrows). DAPI stain (blue) was used to label nuclei. Absence of both CifA and CifB indicates that proteins are not accessible to the antibodies when the sperm chromatin is condensed and tightly packed. 
Fig S5. CifA and CifB are also removed in the cytoplasmic waste bag. Testes $(n=20)$ from $<8$ hrs old males of transgenic $c i f A B$, and wild type $w$ Mel- lines were dissected and immunostained to visualize CifA (green) and CifB (red) in the cytoplasmic waste bags (WB) that are present near the basal end of sperm tail bundles. Some of the Cif proteins strip down in the WB in cifAB line and absent in $w$ Mel- control testes. Brightfield is shown to highlight the morphology of sperm tail bundles and waste bags, which are otherwise not visible using Cif antibodies and DAPI stain. The experiment was run in parallel to the ones shown in Fig 1 and Fig S2.

Fig S6. Full uncropped fluorescent images are shown related to Fig 2A.

\section{Fig S7. Individual CifA- and CifB-expressing lines do not show abnormal histone retention} but are protamine deficient. (A) Testes $(n=15)$ from $<8$ hrs old males of single transgeneexpressing lines cifA, cifB and a non CI-causing control gene WD0508 were dissected to quantify spermatid bundles with histone retention (purple) during late canoe stage of spermatogenesis. DAPI stain (blue) was used to label spermatid nuclei. Total spermatid bundles with DAPI signals and those with retained Histones were manually counted and graphed. Single transgenic expressing lines showed significantly less histones similar to the negative control $w \mathrm{Mel}$ at the late canoe stage. Vertical bars represent mean and error bars represent standard deviation. Letters indicate statistically significant $(\mathrm{p}<0.05)$ differences as determined by multiple comparisons based on a Kruskal-Wallis test and Dunn's multiple test correction. (B) Mature sperms isolated from seminal vesicles $(n=15)$ of $<8 \mathrm{hr}$ old males reared at $21^{\circ} \mathrm{C}$ were stained with fluorescent CMA3 (green) for detection of protamine deficiency in each individual sperm nucleus. Individual sperm head intensity was quantified in ImageJ (see methods) and graphed. cifA- and cifB-expressing lines showed significantly higher fluorescence indicative of reduced levels of protamines compared to wMel- and WD0508 control lines. Vertical bars represent mean and error bars represent standard deviation. Letters indicate statistically significant $(p<0.05)$ differences as determined by multiple comparisons based on a Kruskal-Wallis test and Dunn's multiple test correction. All of the P-values are reported in Table S1. The experiments were performed in parallel to the ones shown in Fig 2. (C) CI hatch rate analyses of transgenic male siblings used in CMA3 assays (Figure 2B, S6) validate that CI crosses (black circles) yielded significant less embryonic hatching compared to non CI-inducing ones, when reared at $21^{\circ} \mathrm{C}$. Letters to the right indicate statistically significant $(\mathrm{p}<0.05)$ differences as determined by multiple comparisons based on a Kruskal-Wallis test and Dunn's multiple test correction. All of the P-values are reported in Table S2. Raw data underlying this figure can be found in S1 data file.

Fig S8. 7 day-old males do not cause CI and are not protamine deficient. (A) Sperms from the 7 day-old wild type Wolbachia-infected ( $w \mathrm{Mel}+)$ males show similar level of protamine levels as of $w$ Mel-. Vertical bars represent mean, and error bars represent standard deviation. Letters indicate statistically significant $(\mathrm{p}<0.05)$ differences as determined by multiple 
923 comparisons based on a Kruskal-Wallis test and Dunn's multiple test correction. All of the Pvalues are reported in Table S1. (B) CI hatch rate analyses of male siblings used in CMA3 assays (panel A) validate that $7 \mathrm{~d}$ old $w \mathrm{Mel}+\mathrm{do}$ not induce $\mathrm{CI}$ that correlates with their normal levels of sperm protamine levels. Letters to the right indicate statistically significant $(p<0.05)$ differences as determined by pairwise Mann-Whitney test and multiple comparisons calculated using a Kruskal-Wallis test and Dunn's multiple test correction. All of the P-values are reported in Table S2. Raw data underlying this figure can be found in S1 data file.

Fig S9. CifA is absent in late oocyte stages and the developing embryos from the rescue cross. (A) In the transgenic cifA line, CifA (green) is absent in the late oocyte stages. Image was acquired at 20x magnification to show mid and late oocytes in one plane. We note the autofluorescence upon enhanced exposure in the green channel outlining the tissue morphology of stage 15 egg chamber does not signify CifA signals. (B) Immunofluorescence of CifA (green) and histones (magenta) in 1-2 hr old embryos $(\mathrm{n}=50)$ obtained from rescue cross (cifAB male $\mathrm{x}$ $w \mathrm{Mel}+$ female). Histone signals are detected in the developing embryos colocalizing with host DNA, labelled with DAPI (blue), whereas CifA signals are absent. 

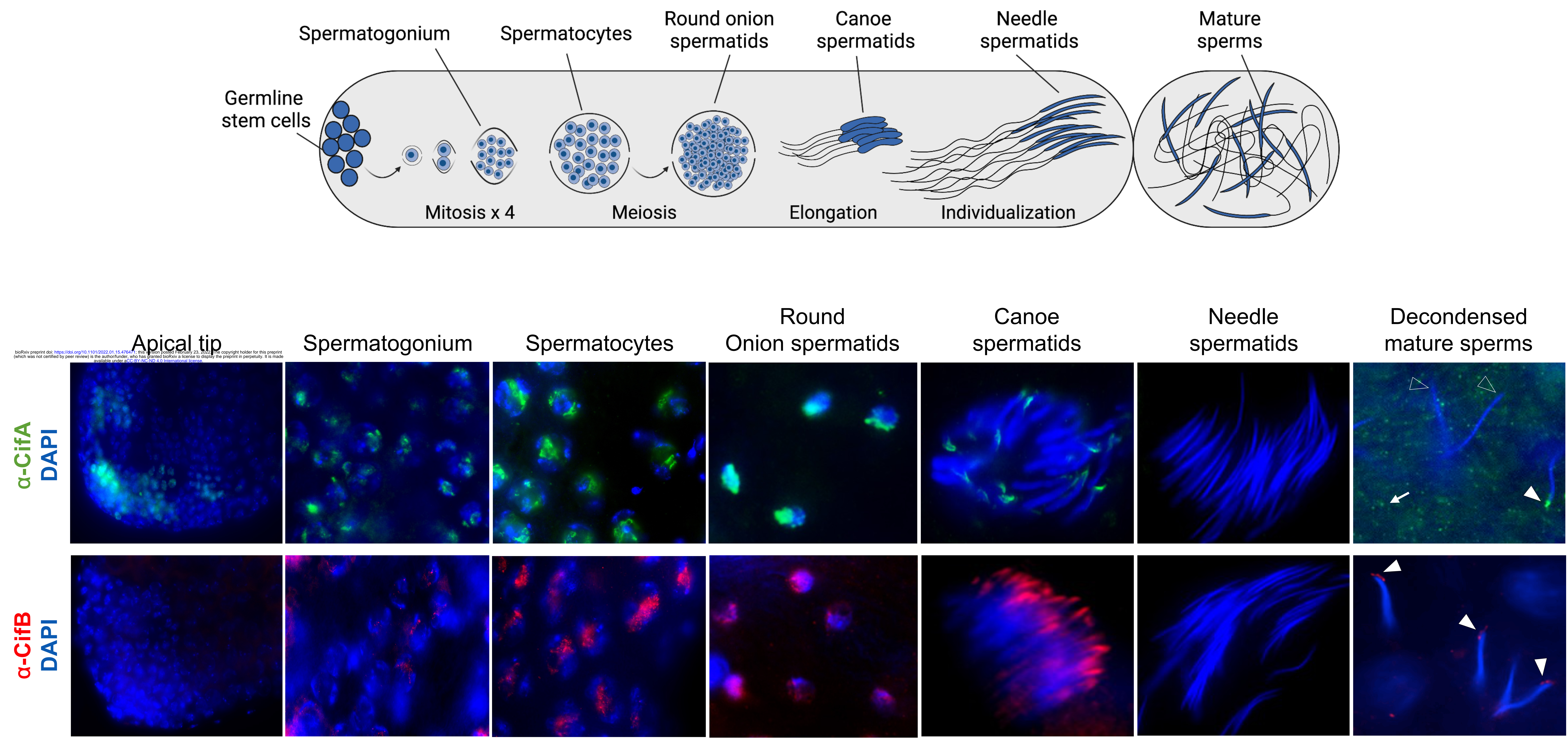
A

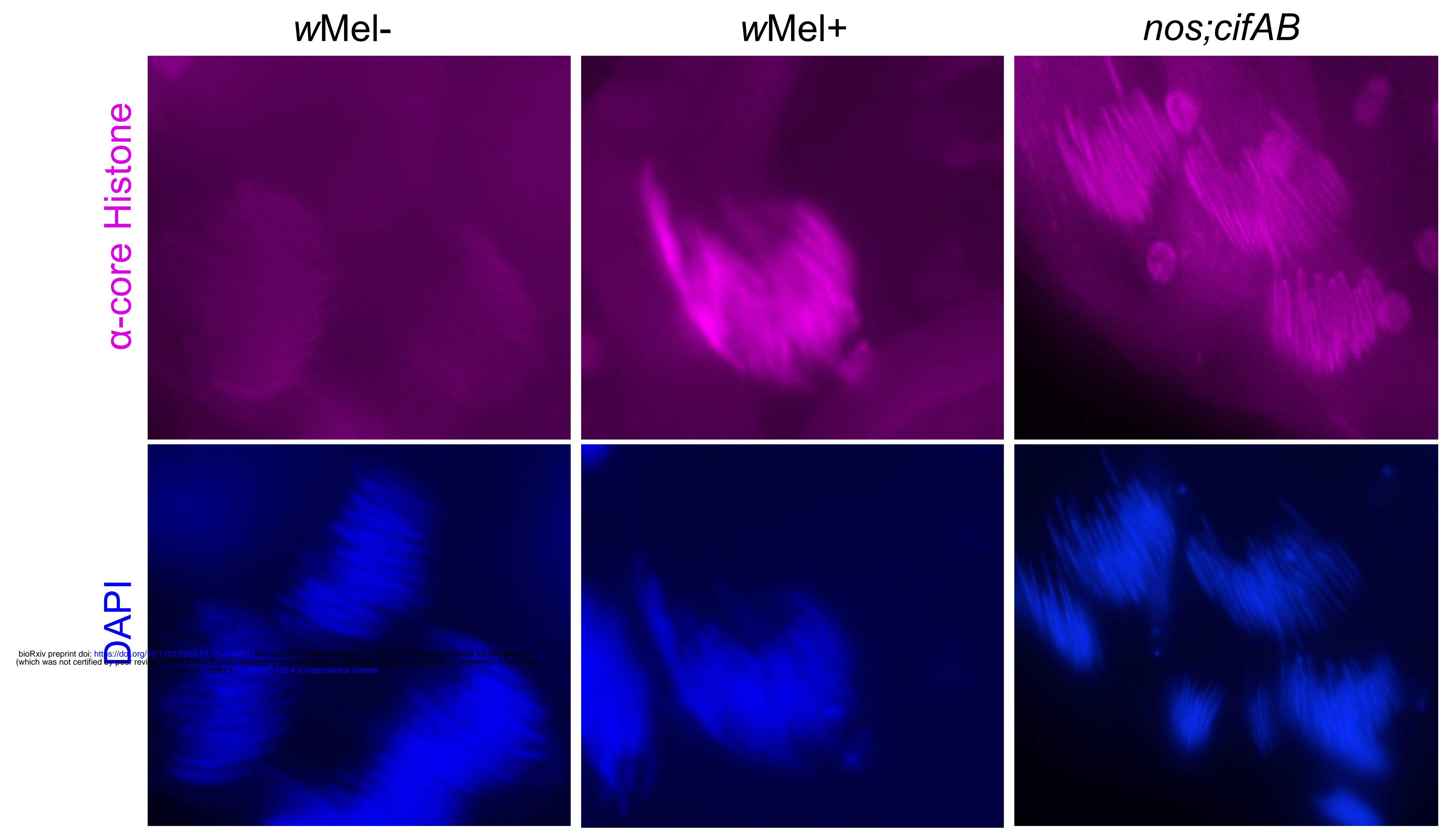

B

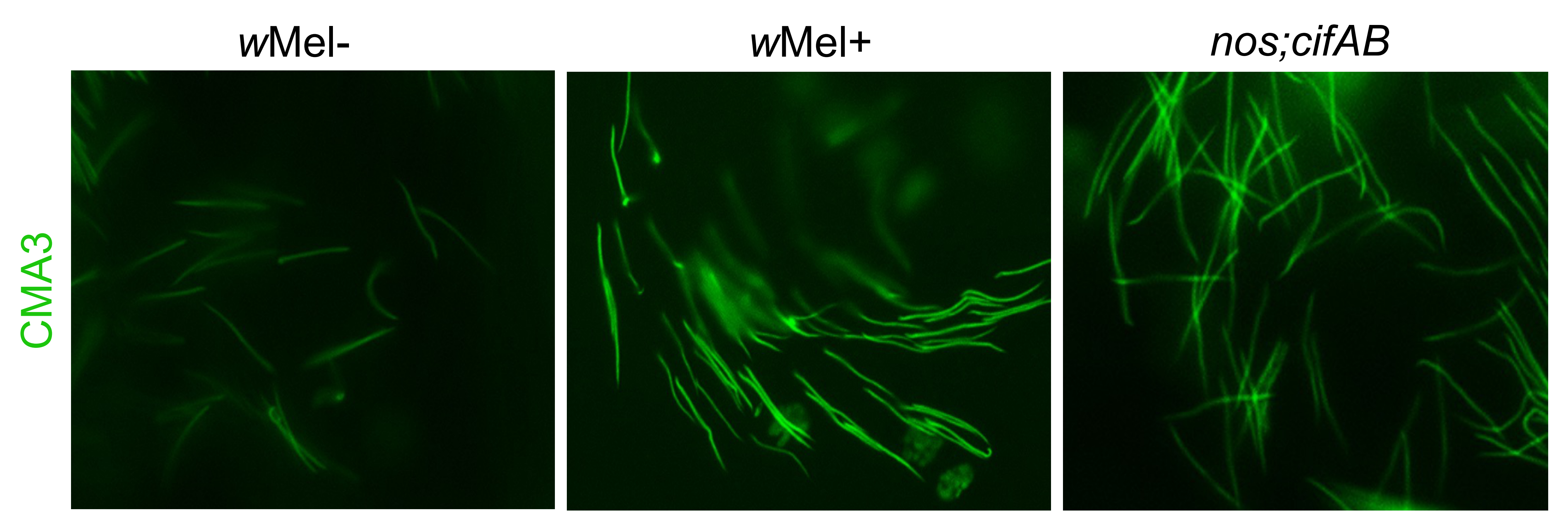

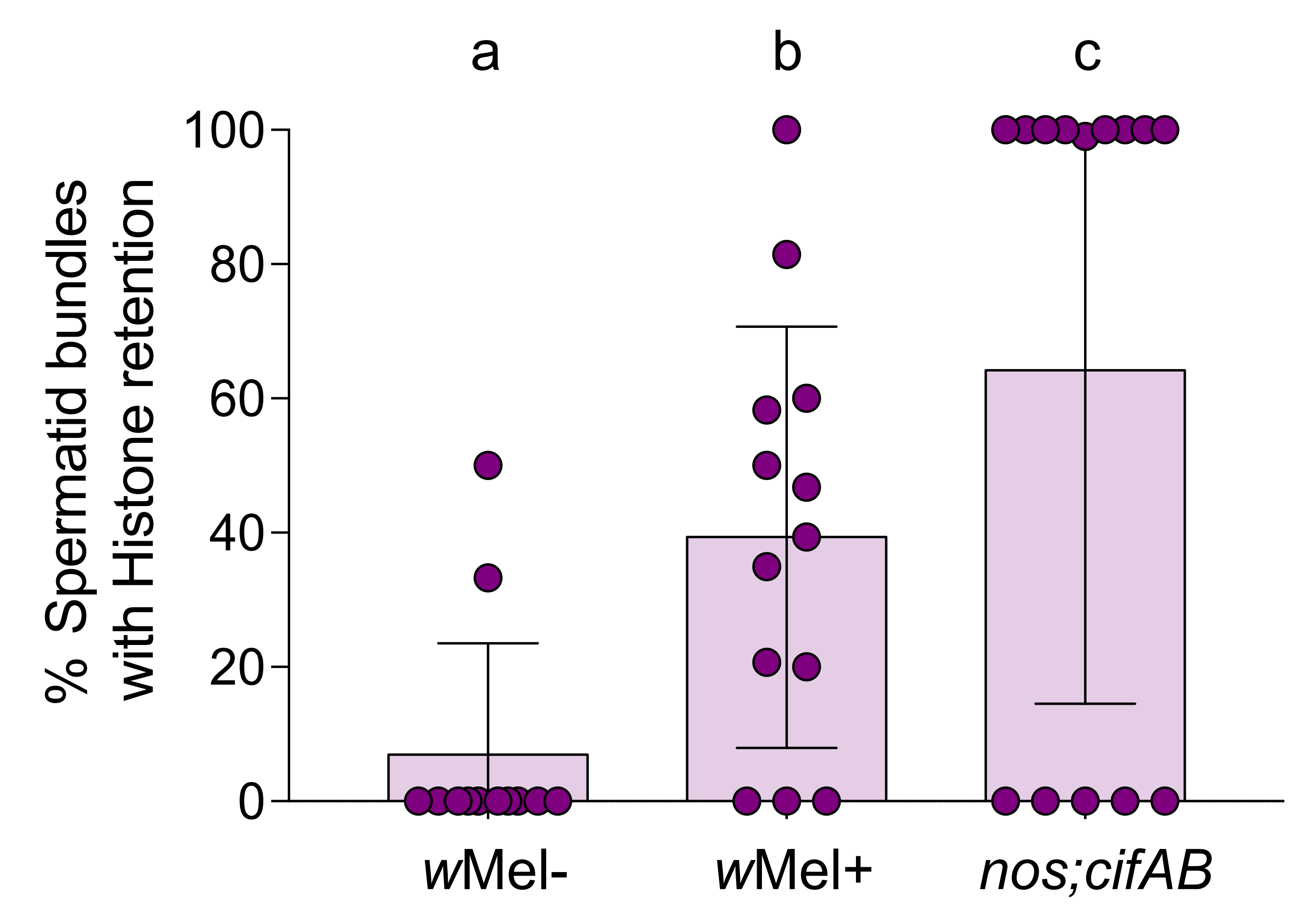

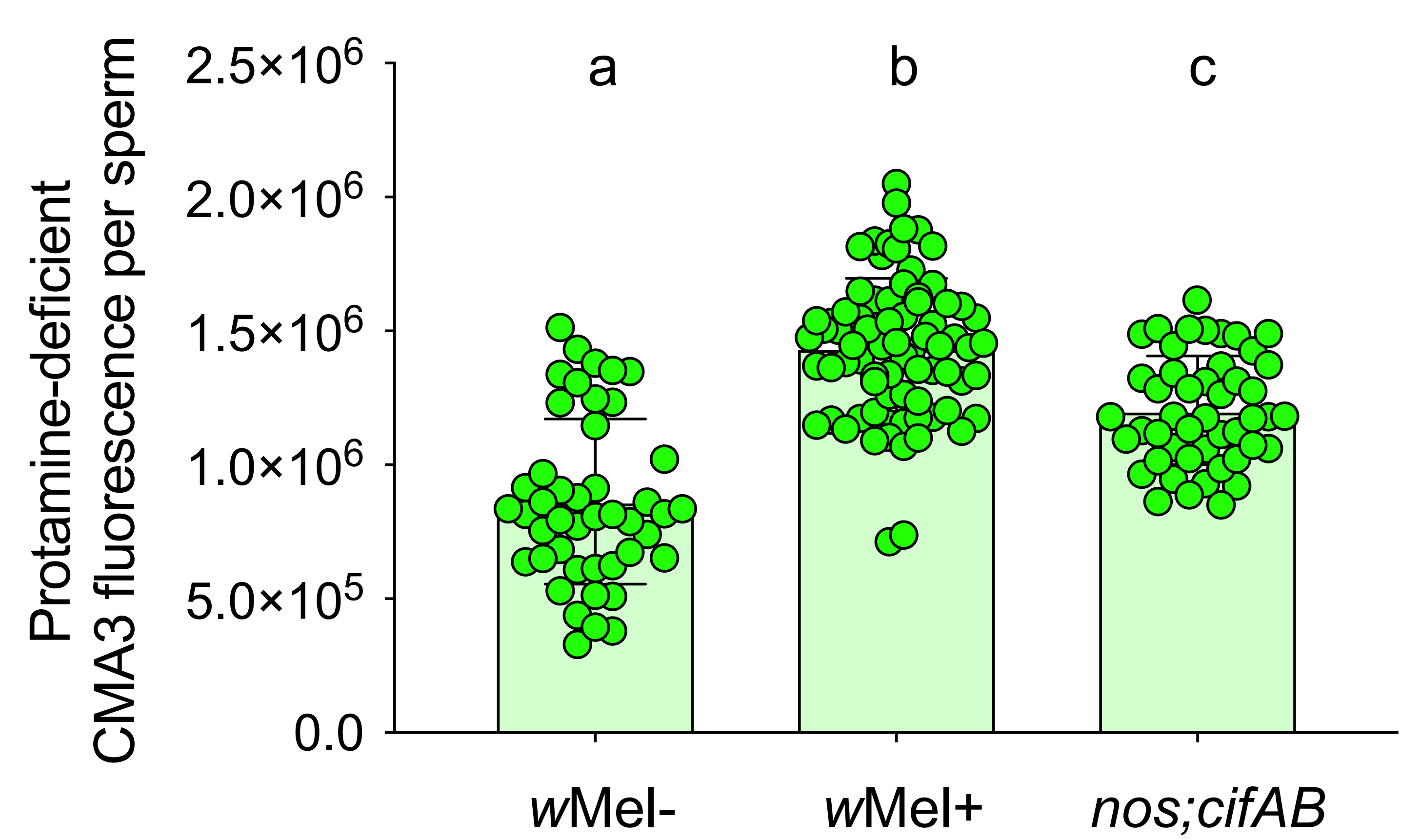


A

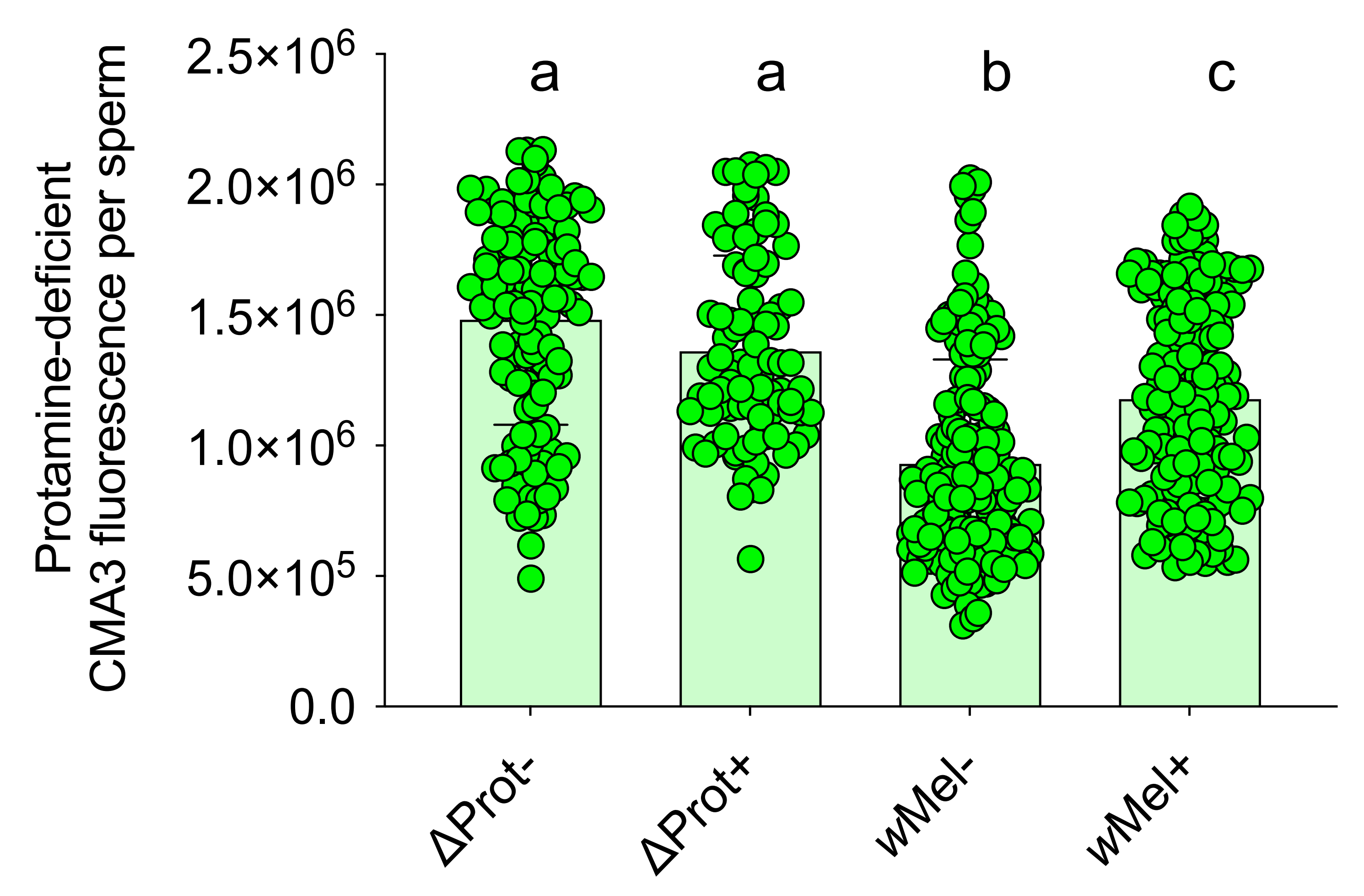

B

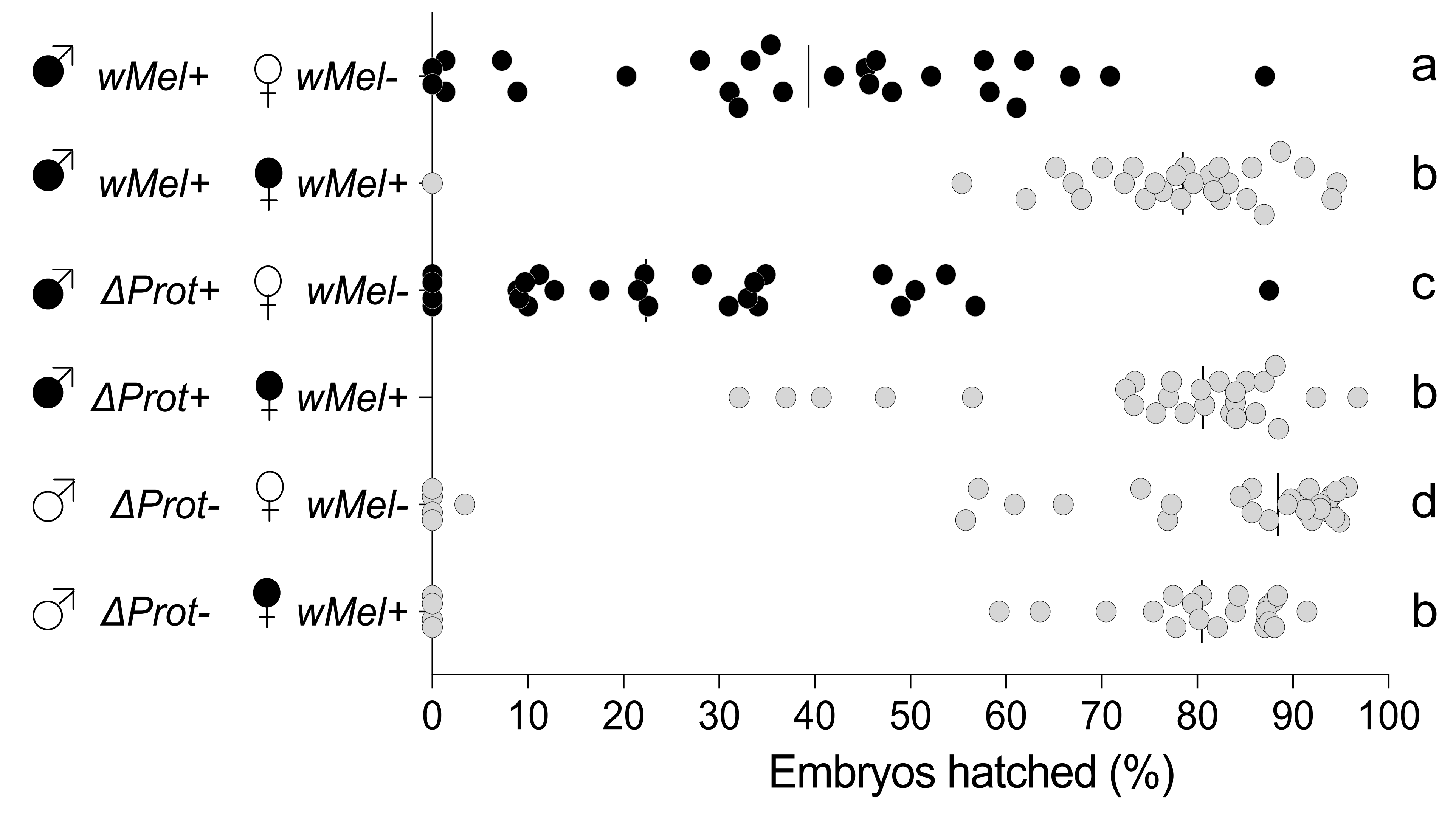


bioRxiv preprint doi: https://doi.org/10.1101/2022.0115.476471: this version posted February 23, 2022. The copyright holder for this preprint (which was not certified by peer review) is the aut 6 dfAlinder, Qhf Ahs granted bioRxiv a license to display the preprint in perpetuity. It is made

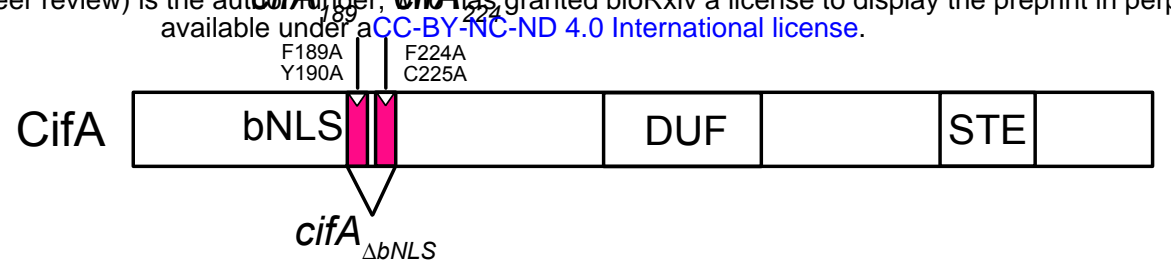

B

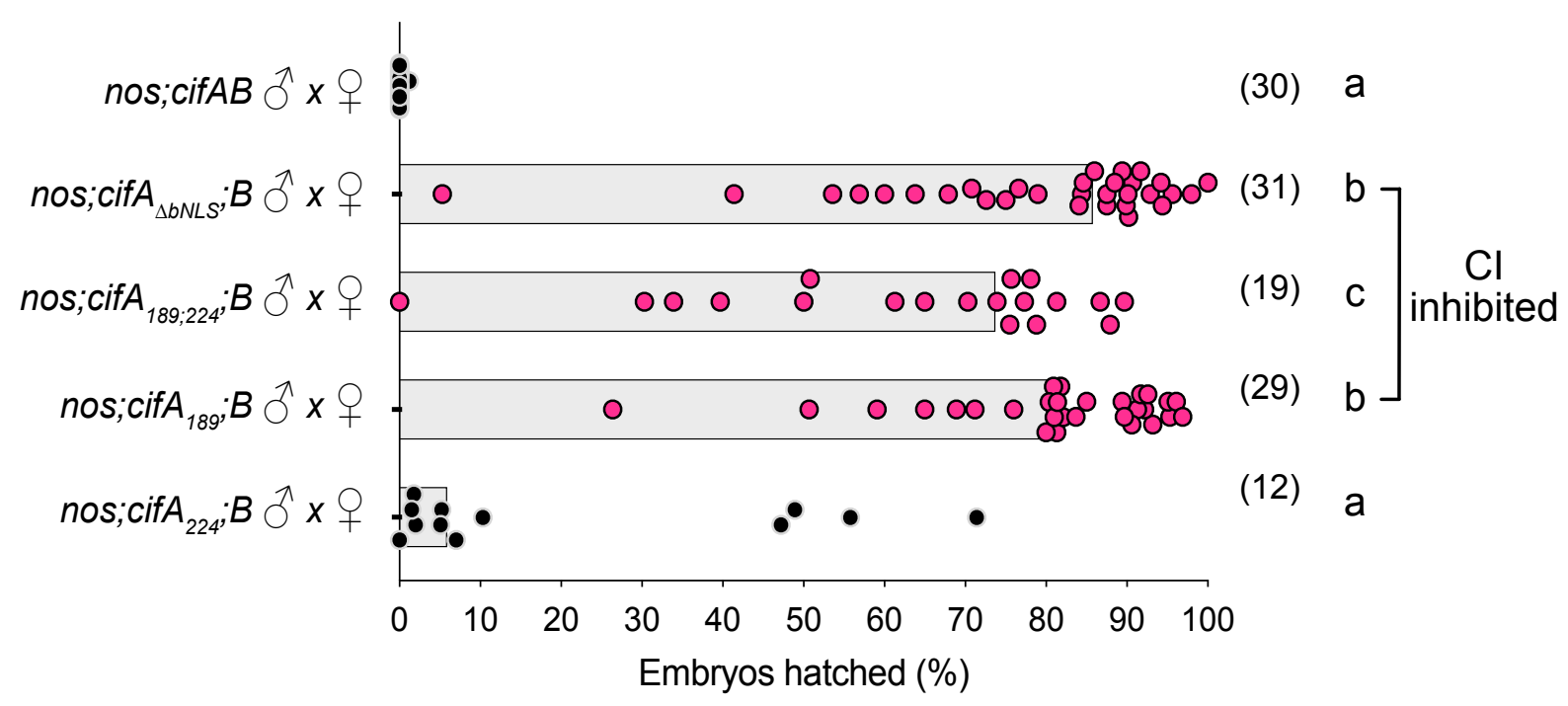

C

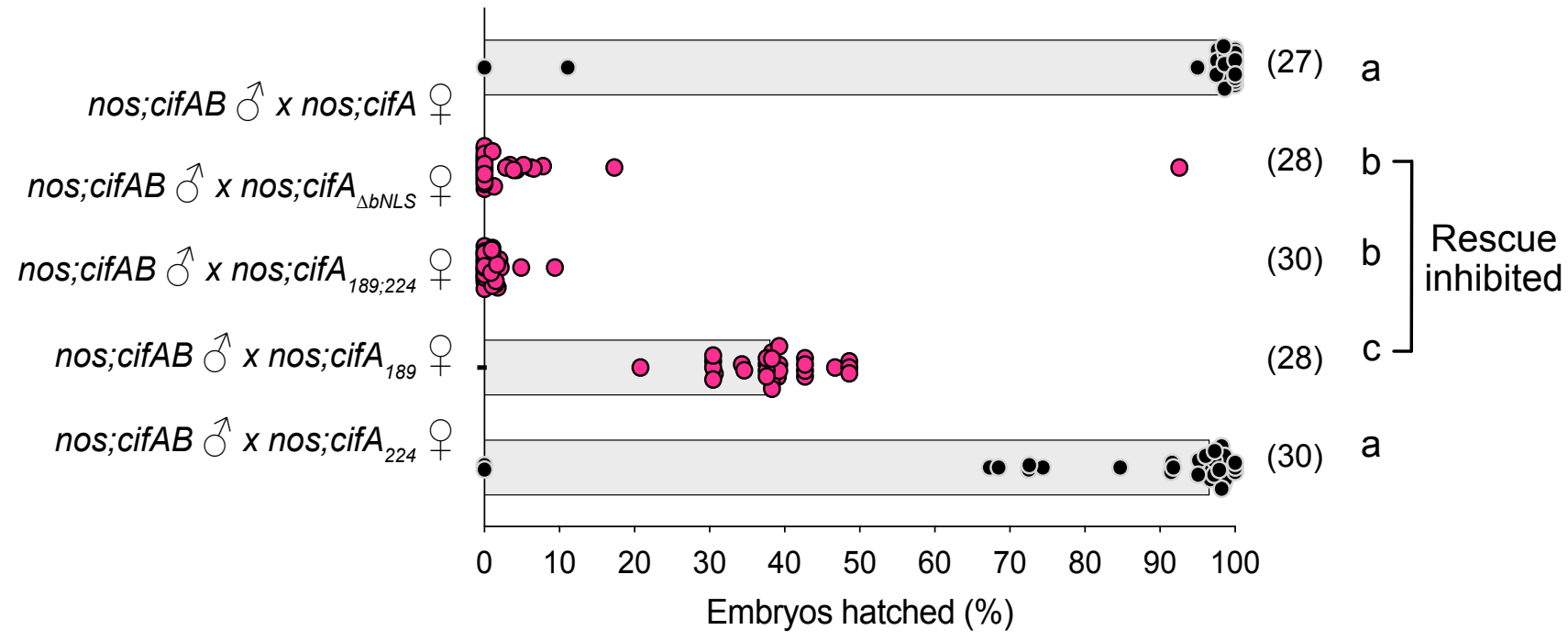

D

E

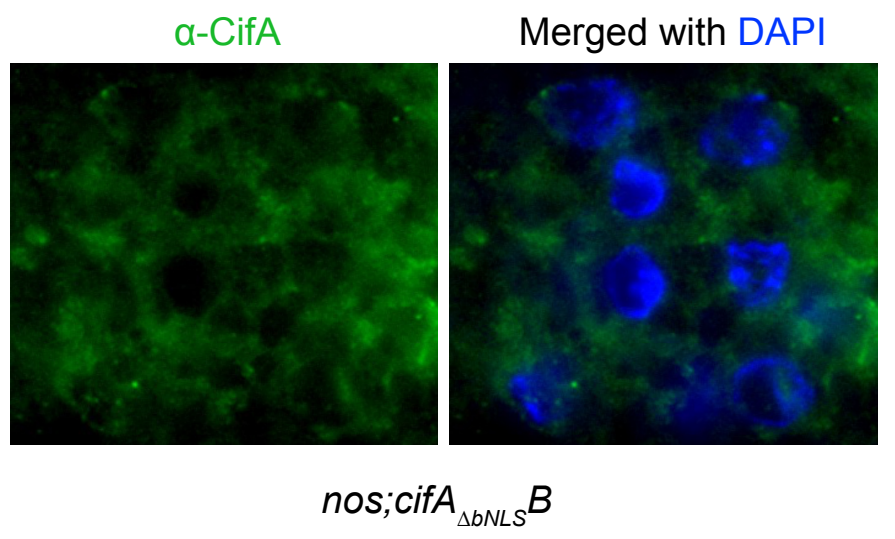

Round onion-stage spermatids

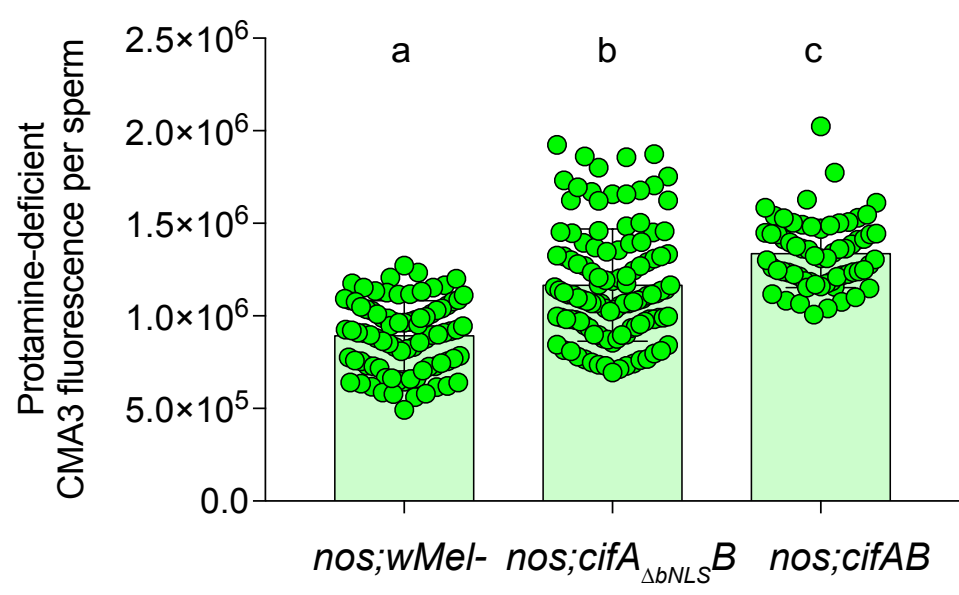


A

B

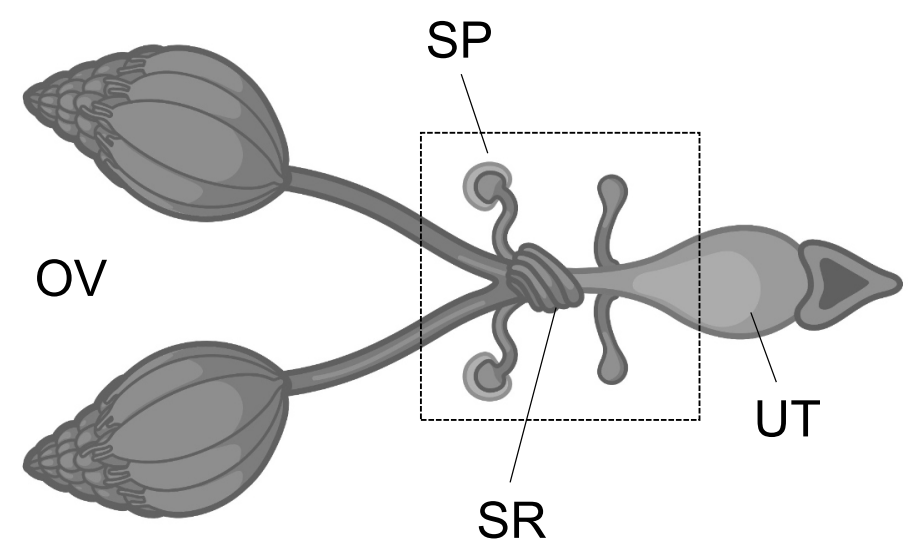

C

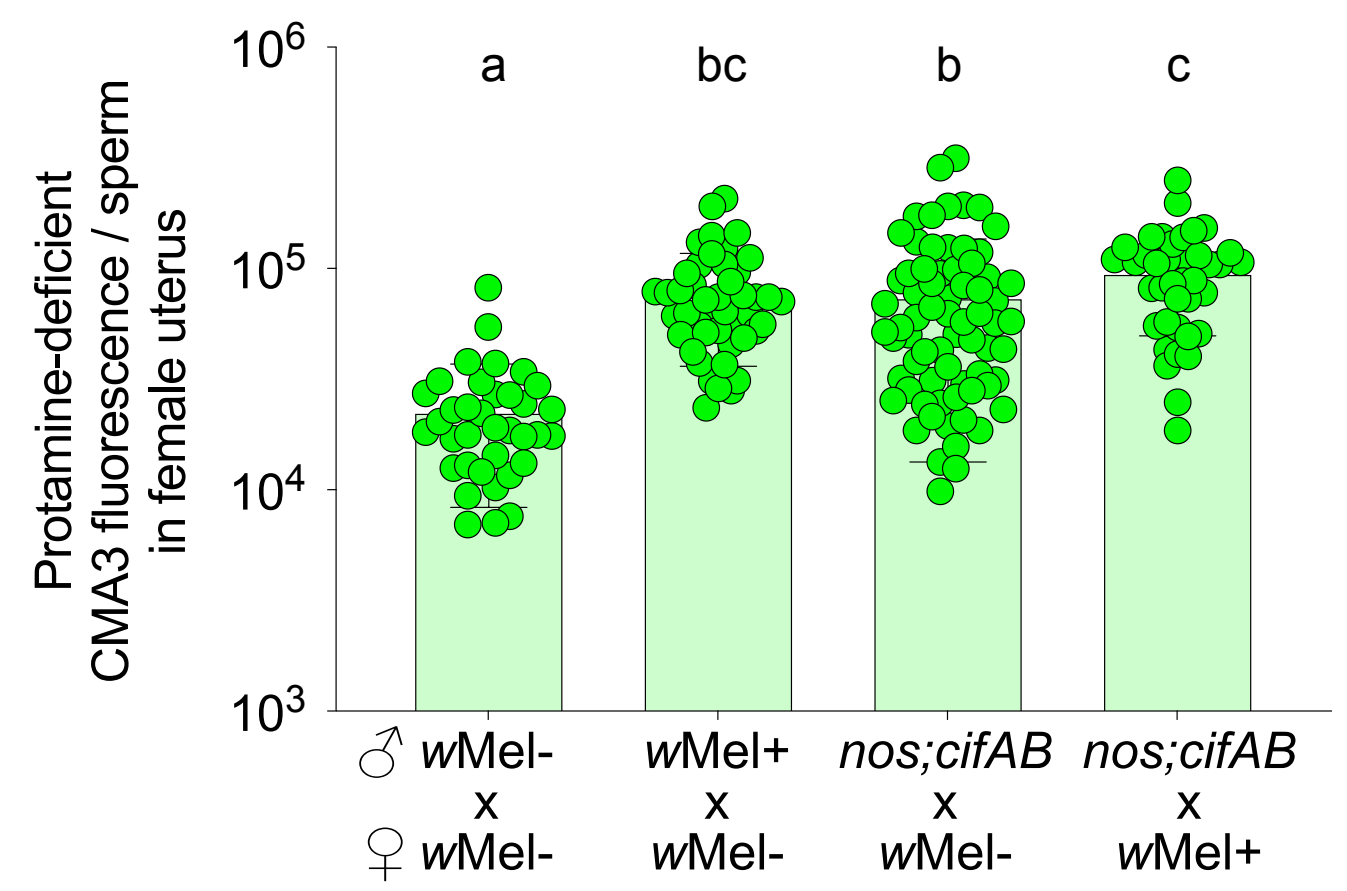

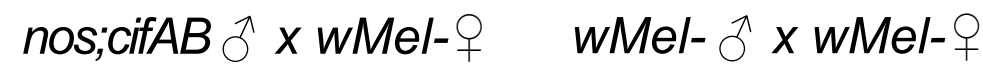

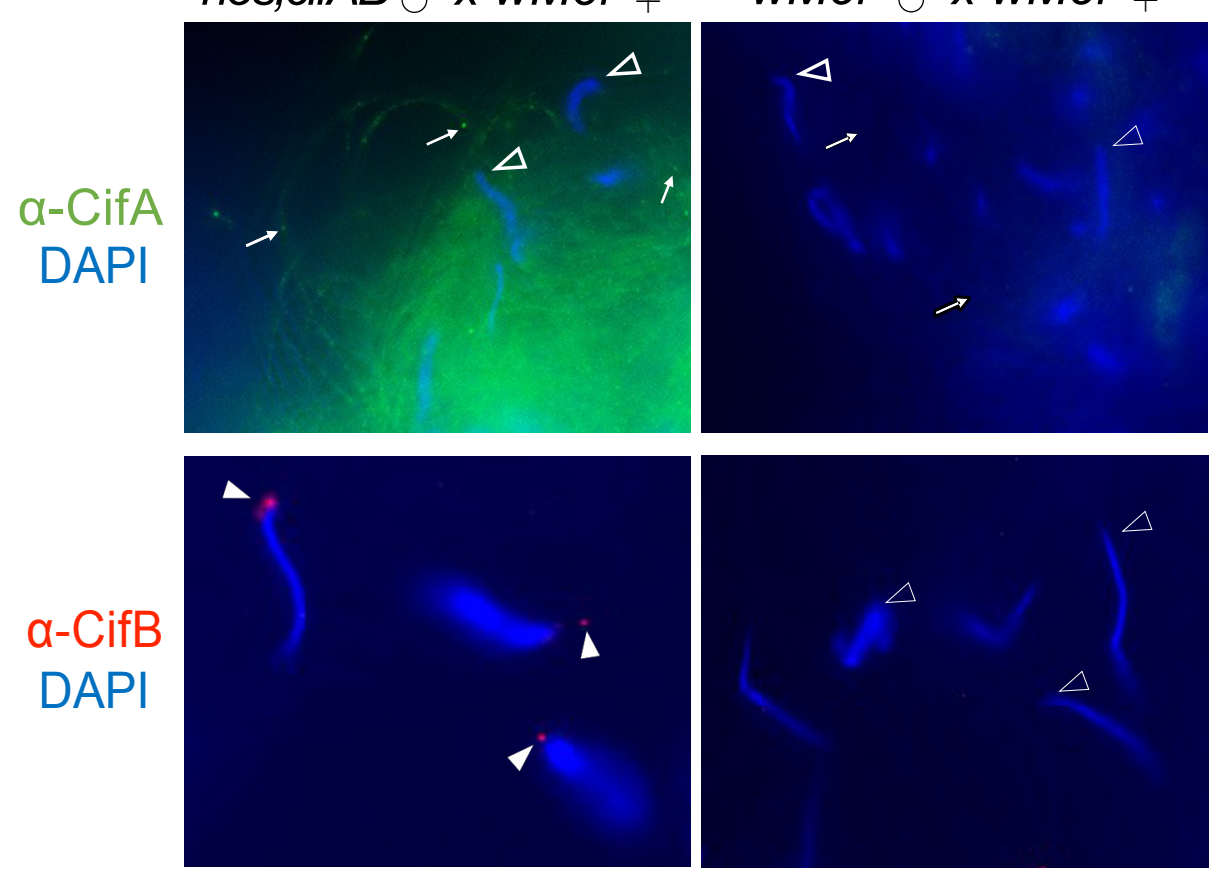

D

wMel- đ̃ x wMel- +

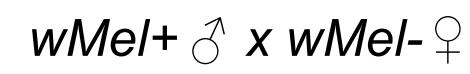

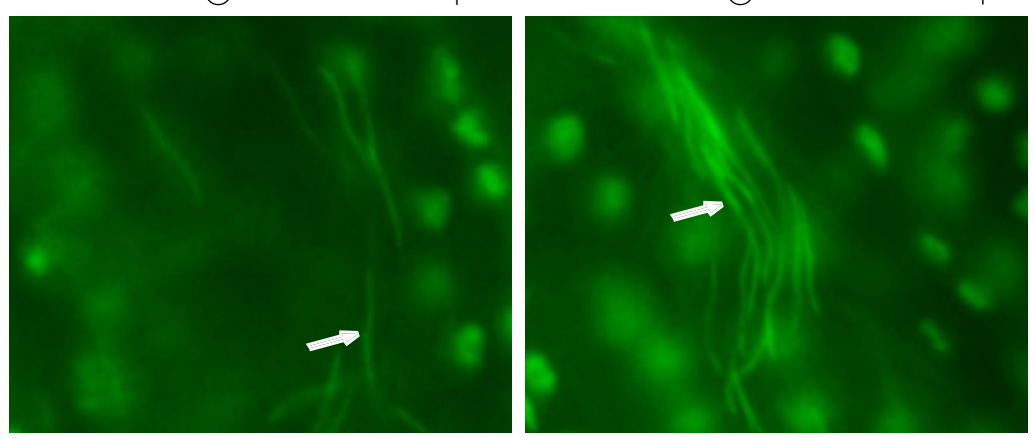

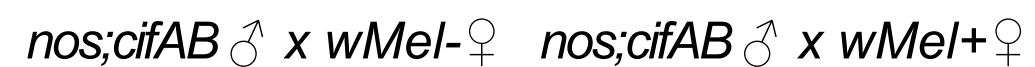

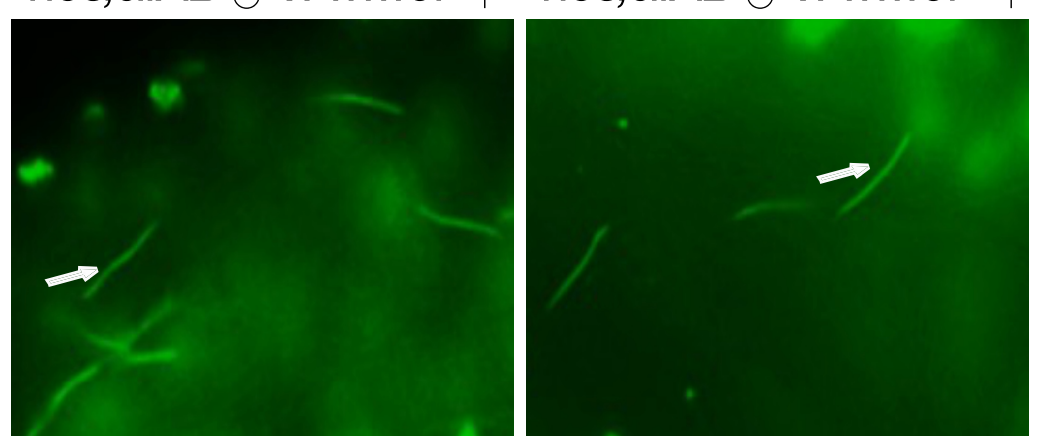


